\title{
Slow Modes of Global Temperature Variability and Their Impact on Climate Sensitivity Estimates
}

\author{
Robert C. J. Wills, ${ }^{a}$ Kyle C. Armour, ${ }^{\mathrm{a}, \mathrm{b}}$ David S. Battisti, ${ }^{\mathrm{a}}$ Cristian Proistosescu, ${ }^{\mathrm{c}}$ And \\ LUKE A. PARSONS ${ }^{\mathrm{d}}$ \\ ${ }^{a}$ Department of Atmospheric Sciences, University of Washington, Seattle, Washington \\ ${ }^{\mathrm{b}}$ School of Oceanography, University of Washington, Seattle, Washington \\ ${ }^{\mathrm{c}}$ Department of Atmospheric Sciences and Department of Geology, University of Illinois, Urbana-Champaign, Illinois \\ ${ }^{\mathrm{d}}$ Nicholas School of the Environment, Duke University, Durham, North Carolina
}

(Manuscript received 30 December 2020, in final form 30 July 2021)

\begin{abstract}
Internal climate variability confounds estimates of the climate response to forcing but offers an opportunity to examine the dynamics controlling Earth's energy budget. This study analyzes the time-evolving impact of modes of low-frequency internal variability on global-mean surface temperature (GMST) and top-of-atmosphere (TOA) radiation in preindustrial control simulations from phase 6 of the Coupled Model Intercomparison Project (CMIP6). The results show that the slow modes of variability with the largest impact on decadal GMST anomalies are focused in high-latitude ocean regions, where they have a minimal impact on global TOA radiation. When these regions warm, positive shortwave cloud and sea ice-albedo feedbacks largely cancel the negative feedback of outgoing longwave radiation, resulting in a weak net radiative feedback. As a consequence of the weak net radiative feedback, less energy is required to sustain these long-lived temperature anomalies. In contrast to these weakly radiating high-latitude modes, El Niño-Southern Oscillation (ENSO) has a large impact on the global energy budget, such that it remains the dominant influence on global TOA radiation out to decadal and longer time scales, despite its primarily interannual time scale. These results show that on decadal and longer time scales, different processes control internal variability in GMST than control internal variability in global TOA radiation. The results are used to quantify the impact of low-frequency internal variability and ENSO on estimates of climate sensitivity from historical GMST and TOA-radiative-imbalance anomalies.
\end{abstract}

KEYWORDS: Climate sensitivity; Climate variability; Energy budget/balance; Feedback; Multidecadal variability

\section{Introduction}

Earth's energy budget forms the basis for understanding changes in global-mean surface temperature (GMST). Energetic constraints suggest that surface temperatures will warm until they give rise to a radiative response that exactly opposes the radiative forcing from an increase in greenhouse gases. In the simple case where the global radiative response depends linearly on the GMST anomaly $\bar{T}(t)$ (e.g., Gregory et al. 2004), the global energy flux imbalance $\bar{N}(t)$ at the top of the atmosphere (TOA) is given by the sum of a global-mean radiative forcing $\bar{F}(t)$ and a global-mean radiative response $\bar{R}(t)=\lambda(t) \bar{T}(t)$ according to

$$
\bar{N}(t)=\bar{F}(t)+\lambda(t) \bar{T}(t) .
$$

Here, overbars denote a global mean and $\lambda(t)$ is the global radiative response per degree of global surface temperature change, called the global climate feedback (Charney et al. 1979; Dessler 2013). The TOA radiative fluxes $\bar{F}(t), \bar{R}(t)$, and $\bar{N}(t)$ are all defined to be positive for downward radiation anomalies.

The global climate feedback $\lambda$ provides the basis for determining Earth's equilibrium climate sensitivity (ECS), the global-mean surface warming that would result from a doubling of atmospheric $\mathrm{CO}_{2}$ (and associated radiative forcing $\bar{F}_{2 \times \mathrm{xO}_{2}} \approx 4 \mathrm{~W} \mathrm{~m}^{-2}$ ) once the climate system has reached equilibrium (Charney et al. 1979):

$$
\mathrm{ECS}=-\frac{\bar{F}_{2 \mathrm{xCO}_{2}}}{\lambda_{\mathrm{eq}}} .
$$

Here, $\lambda_{\text {eq }}$ is the global climate feedback at equilibrium.

Corresponding author: Robert C. Jnglin Wills, rcwills@uw.edu
This energy budget framework has been used to estimate climate sensitivity from observations (Otto et al. 2013; Lewis and Curry 2015; Forster 2016; Knutti et al. 2017; Sherwood et al. 2020). Observational estimates of climate sensitivity rely on diagnosing the global climate feedback $\lambda_{\text {obs }}$ from the observed GMST change $\bar{T}_{\text {obs }}$ and the corresponding radiative response $\bar{R}_{\mathrm{obs}}=\bar{N}_{\mathrm{obs}}-\bar{F}_{\mathrm{obs}}$ :

$$
\text { EffCS }=-\frac{\bar{F}_{2 \mathrm{xCO}_{2}}}{\lambda_{\mathrm{obs}}}=\frac{\bar{F}_{2 \mathrm{xCO}_{2}} \bar{T}_{\mathrm{obs}}}{\bar{F}_{\mathrm{obs}}-\bar{N}_{\mathrm{obs}}} .
$$

EffCS is the effective climate sensitivity corresponding to the feedback $\lambda_{\text {obs }}$. For example, Otto et al. (2013) estimate the climate sensitivity based on decadal anomalies in GMST ( $\left.\bar{T}_{\text {obs }}\right)$, radiative forcing $\left(\bar{F}_{\text {obs }}\right)$, and global ocean heat uptake (an estimate of $\bar{N}_{\text {obs }}$ ) relative to preindustrial values.

The EffCS in observations can differ from the ECS (i.e., $\lambda_{\text {obs }} \neq$ $\lambda_{\text {eq }}$ ) because the radiative response to warming depends not only on GMST but also on the spatial pattern of warming (e.g., Armour et al. 2013; Andrews et al. 2015; Zhou et al. 2017; Dong et al. 2019). Assuming a linear superposition of the radiative feedbacks from warming in different regions, the global climate feedback $\lambda$ can be expressed as

$$
\lambda=\overline{\lambda(\mathbf{r}) T^{\prime}(\mathbf{r})},
$$

where $T^{\prime}(\mathbf{r}) \equiv T(\mathbf{r}) / \bar{T}$ is the local surface warming normalized by the global-mean surface warming (i.e., the spatial pattern of warming) and $\lambda(\mathbf{r}) \equiv \partial \bar{N} / \partial T(\mathbf{r})$ quantifies the global-mean TOA radiative response to local warming $T(\mathbf{r})$.

To constrain $\lambda(\mathbf{r})$, some studies have taken a forward modeling approach, imposing warming in different regions and 
examining the global radiative response (Zhou et al. 2017; Dong et al. 2019). They find that the global radiative response can be reconstructed from the pattern of surface temperature anomalies using a Green's function approach. However, this Green's function depends on the climate model used and is difficult to validate with observations. It is also possible to constrain $\lambda(\mathbf{r})$ by simply regressing global TOA anomalies against local temperature. This has been used to examine the dependence of the global cloud radiative effect on local temperature in climate model simulations and observations (Zhou et al. 2017), but as these authors point out, it does not account for the correlations between temperature anomalies in different regions. Multiple linear regression can be used to account for the correlation between local surface temperature predictors of $\bar{N}$ in climate model output (Bloch-Johnson et al. 2020), but this problem becomes underconstrained when looking at the short observational record.

An alternative approach is to use dimension reduction to determine a few relevant patterns of temperature variability (i.e., modes of variability) and examine their impact on $\bar{T}$ and $\bar{N}$. In the case where a forced climate change can be reconstructed as the sum of changes $\Delta X$ in the indices of all relevant climate modes (cf. fluctuation-dissipation theorem), the success of the Green's function approach used by Zhou et al. (2017) and Dong et al. (2019) suggests that the total global climate feedback could be determined by

$$
\lambda=\frac{\sum_{X}(\partial \bar{N} / \partial X) \Delta X}{\sum_{X}(\partial \bar{T} / \partial X) \Delta X} .
$$

In this way, an analysis of the global temperature and radiative impacts of internal variability can give mechanistic insights into the global climate sensitivity in response to external forcing.

Modes of sea surface temperature (SST) variability such as Atlantic multidecadal variability (AMV), Pacific decadal variability (PDV), and El Niño-Southern Oscillation (ENSO) have all been argued to play leading roles in the decadal modulation of GMST, ocean heat content, and the TOA energy imbalance, such as is thought to have occurred during the so-called global warming hiatus, from 1998 to 2013 (Meehl et al. 2011; Trenberth and Fasullo 2013; Kosaka and Xie 2013; England et al. 2014; Liu et al. 2016). In this way, internal variability can lead to contributions to $\bar{T}_{\text {obs }}$ and $\bar{N}_{\text {obs }}$ that are not representative of the forced response, confounding estimates of the climate response to external forcing based on Eq. (3). However, there is no consensus on the relative importance of these different modes for variability in global climate. Some studies emphasize the importance of processes in the eastern equatorial Pacific (Meehl et al. 2011, 2013; Kosaka and Xie 2013; Trenberth and Fasullo 2013; England et al. 2014; Risbey et al. 2014; Liu et al. 2016), while others emphasize processes in the North Atlantic (Zhang et al. 2007; Keenlyside et al. 2008; DelSole et al. 2011; Muller et al. 2013; Chen and Tung 2014; Tung et al. 2018; Stolpe et al. 2018; Li et al. 2020) or the Southern Ocean (Martin et al. 2013; Latif et al. 2013; Brown et al. 2015; Cabré et al. 2017). These different conclusions arise in part because different models emphasize processes in different regions (Brown et al. 2015; Parsons and Hakim 2019; Parsons et al. 2020) and in part because the relationships between local temperature, global temperature, and global TOA radiation depend on the time scale of variability (Brown et al. 2015; Lutsko and Takahashi 2018).

In this paper, we quantify the impact of modes of lowfrequency (interdecadal) SST variability on GMST and the global-mean TOA radiation flux (GMTOA) in coupled climate models. Traditional indices of low-frequency variability (e.g., PDV, AMV, etc.) are problematic for this analysis for a number of reasons. For one, they are not in general independent of each other. This by itself could be circumvented with multiple linear regression. However, traditional indices are also highly sensitive to subtleties of their definitions (Frankcombe et al. 2015; Stolpe et al. 2017) and have been shown to mix together physically distinct processes that may have different impacts on global climate (Newman et al. 2016; Wills et al. 2019a,b). Principal component analysis provides a promising candidate for defining independent climate indices, but it also has the tendency to mix together processes that occur on different time scales and in different regions (Chen and Wallace 2016; Chen and Tung 2017; Wills et al. 2018). We therefore use low-frequency component analysis (LFCA; Wills et al. 2018) to identify modes of interdecadal variability. This method identifies linear combinations of principal components that isolate variability at decadal and longer time scales. We use lead-lag regressions of GMST and GMTOA anomalies onto these slow modes of variability to quantify their timeevolving impacts on global climate and their influence on EffCS. We also contrast the global climate impacts of interdecadal variability with those of interannual ENSO variability, because ENSO influences GMST and the global energy budget across a wide range of time scales.

This paper is organized as follows. We describe the CMIP6 output and methods used in section 2. In section 3, we describe the slow modes of internal variability in CMIP6 models and quantify their impacts on GMST and GMTOA. We also quantify the impacts of ENSO on decadal variability in GMST and GMTOA for comparison. In section 4, we diagnose the local shortwave and longwave components of GMTOA variability and discuss the mechanisms governing the slow modes of climate variability and their impacts on GMTOA. In section 5, we quantify the impact of modes of internal variability on estimates of climate sensitivity and discuss the future work needed to apply this model-based understanding toward attributing observed changes in Earth's energy budget. In section 6, we summarize our findings and present our conclusions.

\section{Methods and climate model output}

\section{a. Low-frequency component analysis}

Wills et al. (2018, hereafter W18) demonstrate a statistical methodology - low-frequency component analysis (LFCA) — to identify characteristic spatial anomaly patterns of low-frequency variability. LFCA is based on linear discriminant analysis, which has been used for a number of other applications within climate science (Déqué 1988; Schneider and Griffies 1999; Venzke et al. 1999; Schneider and Held 2001; DelSole 2001; Ting et al. 2009; DelSole et al. 2011; Wills et al. 2020). W18 show that LFCA can 
separate the influences of global warming, PDV, and ENSO on Pacific SSTs based on differences in their spatial patterns and time scales. Subsequent papers (Wills et al. 2019a,b; Oldenburg et al. 2021) have shown that LFCA isolates the processes controlling low-frequency variability from those that are important at higher frequencies, for example isolating the role of ocean circulation changes in AMV and PDV.

LFCA solves for low-frequency patterns (LFPs), which are the linear combinations of a chosen set of empirical orthogonal functions (EOFs) that maximize the ratio of low-frequency to total variance in their corresponding time series [low-frequency components (LFCs)]. Here, we define low-frequency variance as that which makes it through a 10-yr low-pass filter. In this way, patterns of interdecadal variability show up as the leading LFPs, and LFP-1 is the linear combination of the included EOFs with the highest possible ratio of interdecadal-to-intradecadal variance. LFPs and LFCs are normalized such that the LFP shows the anomaly pattern corresponding to a one-standard-deviation anomaly in the corresponding LFC, which has unit variance. The LFCs are mutually uncorrelated, but there can be pattern correlation between the LFPs. The main hyperparameter in this analysis is the number $n$ of EOFs retained. W18 explores the sensitivity to $n$ for short ( $\sim 100$ yr) observational datasets; however, we find that our results are insensitive to $50 \leq n \leq 500$ for the large multimodel dataset analyzed here, where the leading $\sim 180$ EOFs are well sampled according to the North et al. (1982) criterion.

By first solving for the EOFs of the unfiltered data, LFCA uses information about the spatiotemporal covariance of both low-frequency and high-frequency variability (i.e., the leading LFC has the highest ratio of low-frequency to high-frequency variance, whereas the leading principal component of low-pass filtered data would have the highest total low-frequency variance). Patterns of intradecadal variability (such as ENSO) therefore show up as the trailing LFCs. In this way, LFCA provides a new basis for the variability contained within the $n$ leading EOFs that is sorted by time scale. We also analyze the interannual ENSO signal captured by the least-low-frequency component (LLFC), which is the $n$th LFC (i.e., the linear combination of the included EOFs with the lowest possible ratio of interdecadal-to-intradecadal variance).

\section{b. CMIP6 preindustrial control output}

We analyze output from preindustrial control (piControl) simulations of 35 coupled climate models from CMIP6 (Eyring et al. 2016). External forcing from greenhouse gasses, aerosols, ozone, and solar variability is fixed at preindustrial levels throughout the simulations. We use the last 400 years of monthly output from each model's piControl simulations such that we include a total of 14000 years of unforced variability in our analysis. For each model, output is quadratically detrended and linearly interpolated to a common $2^{\circ}$ analysis grid. SSTs are computed from surface temperature output by setting all monthly values below the freezing point of seawater to the freezing point and masking out land. This avoids interpolating from each model's irregular ocean grid. GMST anomalies are computed from surface temperature. TOA radiation anomalies are computed from the outgoing longwave radiation and reflected shortwave radiation, because incoming shortwave radiation does not vary about its climatological seasonal cycle in these simulations.
We compute anomalies with respect to each model's climatological seasonal cycle such that intermodel differences in climatology are not included in the analysis. We concatenate each model's SST anomaly matrix into one large anomaly matrix for the multimodel ensemble. LFCA is then used to compute the leading LFPs of global SST anomalies across all 35 piControl simulations. We retain $n=65$ EOFs to capture $63 \%$ of the total SST variance. The $10-y r$ low-pass filter (used to find the LFPs) is applied to each model separately to avoid filtering over the discontinuities between models.

Like principal component analysis, LFCA can mix together processes occurring in different regions. This is especially true for patterns with similar eigenvalues (i.e., similar ratios of interdecadalto-intradecadal variance). For LFPs with similar eigenvalues, we apply a secondary rotation of the resulting LFPs in order to spatially localize them (cf. Kaiser 1958). In this rotation, the pattern correlation between LFPs is computed in specified regions: the Atlantic $\left(45^{\circ} \mathrm{S}-80^{\circ} \mathrm{N}\right)$, the North Pacific $\left(20^{\circ}-70^{\circ} \mathrm{N}\right)$, and the Southern Ocean $\left(35^{\circ}-80^{\circ} \mathrm{S}\right)$. The pattern correlations between a pair of LFPs within each region make up one element of a matrix, the eigenvectors of which give linear combination coefficients that define the new rotated patterns. This rotation is applied separately for sets of LFPs for which the variance ratio is similar (LFPs 4-6 in our case). For example, the rotated LFP-4' is a linear combination of the original LFPs 4-6, where primes denote a rotated pattern. No rotation is needed for LFPs 1-3, because these patterns have distinct ratios of low-frequency to total variance in our particular analysis.

\section{c. Lead-lag regression analysis}

The assessment of the impact of internal variability on global climate is complicated by the phase lags between GMST, GMTOA, and modes of internal variability (Brown et al. 2014; Xie et al. 2016; Proistosescu et al. 2018; Lutsko and Takahashi 2018). In this study, we take the simple approach of computing a lead-lag regression of GMST and GMTOA against each index of internal climate variability. This allows us to show the time-evolving impact of each mode of variability on global climate (e.g., Fig. 1). The time evolution of GMST and GMTOA impacts results primarily from changes in the SST anomaly pattern over time, rather than from lead-lag responses to a fixed SST pattern. For examples of the time evolution of the SST patterns, see Fig. 6 of Wills et al. (2019a) and Fig. 4 of Wills et al. (2019b). It is important to note that the LFCs are only uncorrelated at lag 0 , and lag 0 is therefore the only lag where impacts on GMST and GMTOA are additive. An alternative approach would be to compute the cross spectra of GMST and GMTOA with each mode of variability. However, by allowing for different phase lags between GMST/GMTOA and the LFC at each frequency, this method would not take advantage of the orthogonality of the LFCs at lag 0 and would complicate an assessment of the relative contributions of different modes of low-frequency variability to global climate anomalies.

\section{Slow modes of SST variability and their impact on global climate}

The first six LFPs of the CMIP6 ensemble are shown in Fig. 1. LFP-1 and LFP-2 both show low-frequency variability of SST in the high-latitude oceans, with LFP-1 focused in the 

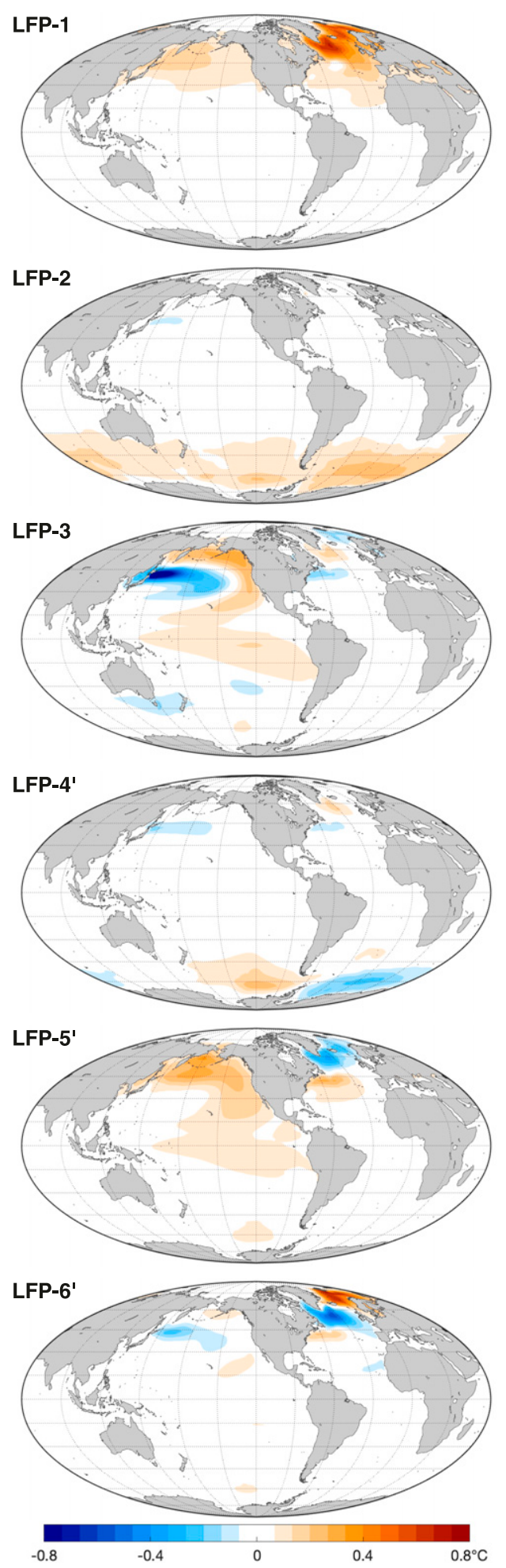

FIG. 1. Low-frequency patterns (LFPs) 1-6 of CMIP6 piControl simulations and their impacts on GMST and GMTOA. (left) The SST anomaly pattern (LFP). (center) The lead-lag regression of monthly GMST anomalies on the associated low-frequency components (LFCs). (right) The lead-lag regression of monthly GMTOA anomalies on the associated LFCs; negative values indicate a loss of energy to space. Black lines show the same lead-lag regressions, but for the 10-yr running mean GMST and GMTOA anomalies. Positive lags indicate anomalies that occur after the maximum anomaly in the LFC. All calculations are averaged over 35 CMIP6 models. A secondary rotation has been applied to LFPs 4-6 in order to localize them within ocean basins, as described in section 2 b.
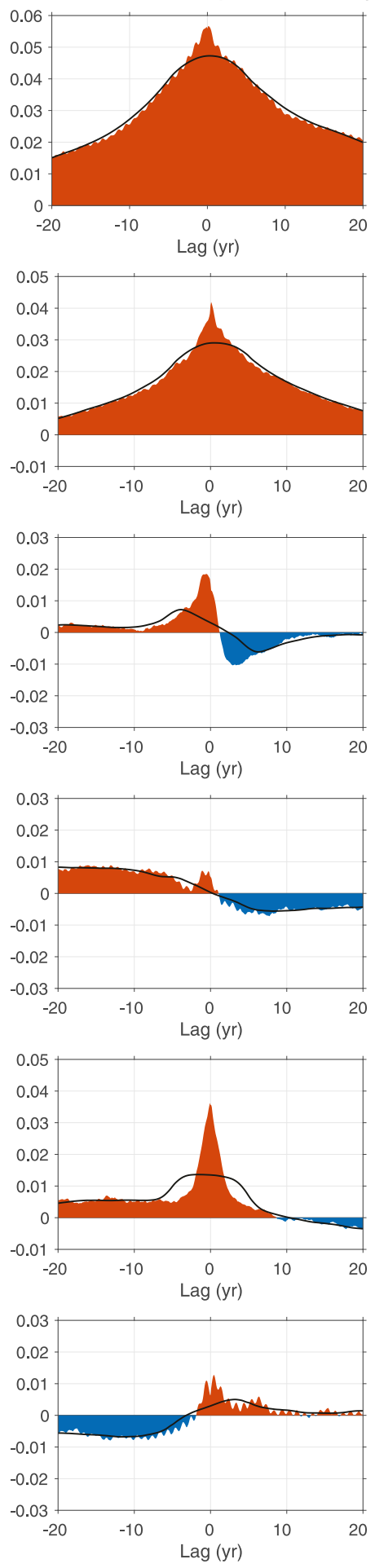

Global-Mean Surface Temperature Anomaly $\left({ }^{\circ} \mathrm{C}\right)$
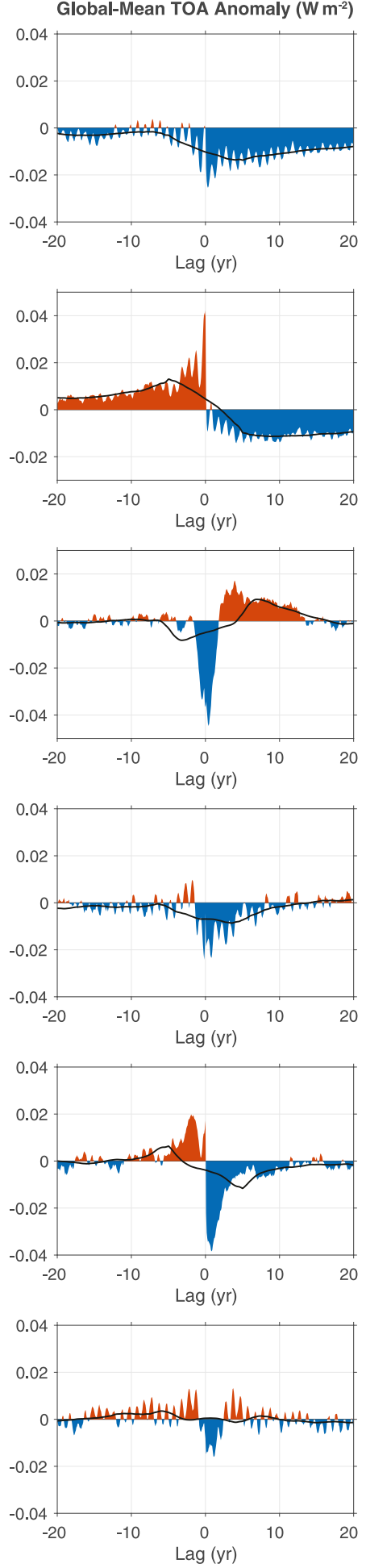

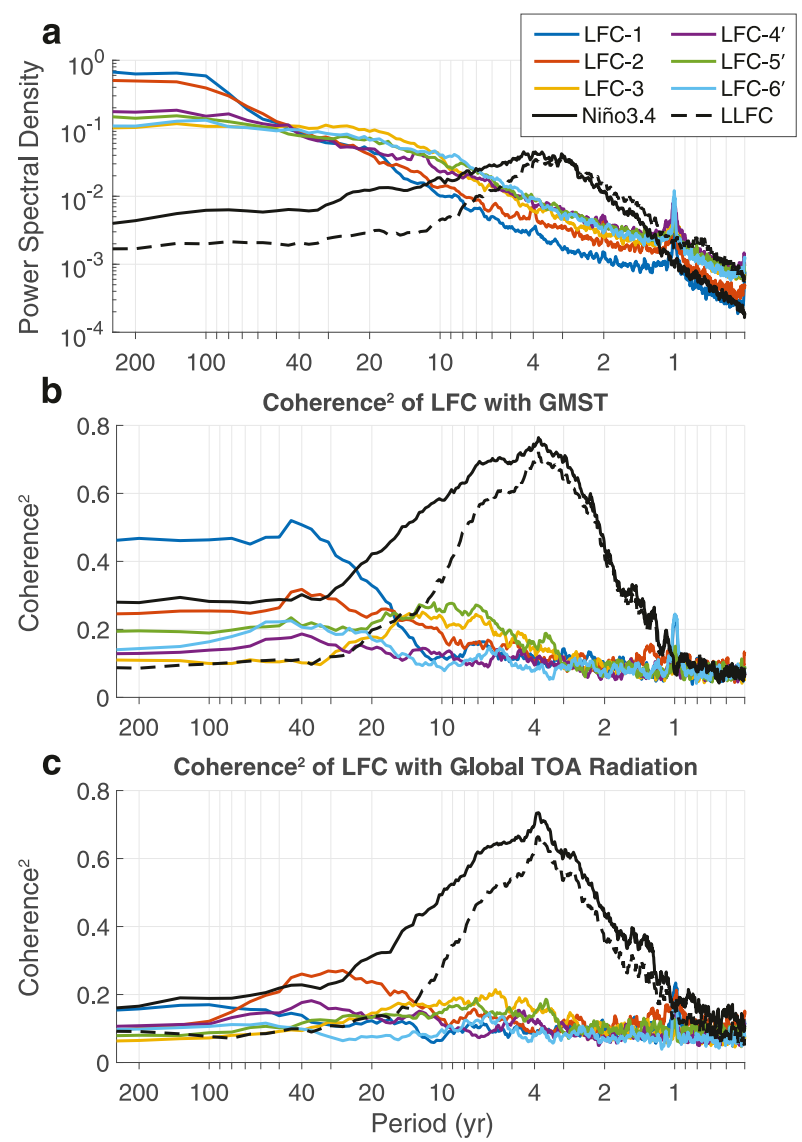

FIG. 2. (a) Power spectral density of the low-frequency components (LFCs). (b) Squared coherence between the LFCs and GMST. (c) Squared coherence between the LFCs and GMTOA. All panels also show Niño-3.4 and the least-low-frequency component (LLFC) for comparison. The LLFC is strongly correlated with Niño-3.4 and is discussed in more detail in the text. All calculations are averaged over 35 CMIP6 piControl simulations.

North Atlantic and LFP-2 focused in the Southern Ocean. LFP-1 is similar to the AMV-like pattern found by LFCA of Atlantic SSTs (Wills et al. 2019a), with a temporal correlation of 0.94 , and is also highly coherent $(0.89)$ with the traditional AMV index (Zhang et al. 2019) on decadal and longer time scales. LFP-2 is similar to the leading LFP of Southern Ocean SSTs when they are analyzed separately (not shown). LFP-3 is similar to the PDV; in particular, it has a spatial pattern similar to the PDV in observations (Mantua et al. 1997), a high coherence (0.96) with the traditional PDV index (Mantua et al. 1997) on decadal and longer time scales, and a temporal correlation of 0.87 with the LFC-PDV index defined by LFCA of Pacific SSTs (Wills et al. 2019b). We find similar patterns of low-frequency internal variability in CMIP5 piControl simulations (not shown). While these LFPs explain less of the total SST variance than the leading EOFs $(2.0 \%, 1.1 \%$, and $2.1 \%$, respectively, vs. $11 \%$, $3.3 \%$, and $2.6 \%$ for the leading EOFs), they explain more of the SST variance on decadal and longer time scales $(11 \%, 5.0 \%$, and $7.0 \%$, respectively, vs. $8.9 \%, 5.0 \%$, and $2.8 \%$ for the leading EOFs). Note that the LFPs show the SST pattern associated with the corresponding LFCs at lag 0, and lead-lag regressions of SST anomalies onto the LFCs show time-evolving SST patterns (Wills et al. 2019a,b).

The next three LFPs show secondary modes of low-frequency SST variability in the Southern Ocean, Pacific, and North Atlantic, respectively. Because they all have similar ratios of interdecadal-to-intradecadal variance, a secondary rotation was applied to localize them spatially (see section $2 b$ ). LFP-4' shows an SST anomaly dipole between the Weddell and AmundsenBellingshausen Seas, a mode of variability identified (in GFDL CM2.1) by Zhang et al. (2017). LFP-5' shows warming of the North Pacific, with some extension into the tropical Pacific along the path of the Pacific meridional mode (Chiang and Vimont 2004). It resembles the Victoria Mode (Bond et al. 2003), which is similar to the SST signature of the North Pacific Gyre Oscillation (NPGO; Di Lorenzo et al. 2008). It also shows a concurrent SST anomaly dipole in the subpolar North Atlantic. LFC- $6^{\prime}$ is similar to the second LFP of the Atlantic-only analysis of Wills et al. (2019a), showing an SST anomaly in the subpolar gyre and opposite signed SST anomalies in the Gulf Stream, Greenland-Iceland-Norwegian (GIN) Seas, and Barents Sea, a well-known mode of Atlantic decadal variability (e.g., Menary et al. 2015). Several modes identified by our analysis show midlatitude SST anomalies in both the Atlantic and Pacific, possibly related to interbasin teleconnections (e.g., Zhang and Delworth 2007). However, such interbasin relationships should be interpreted with caution, as spatial covariance analyses such as this one are known to show coherent anomalies in regions that are only weakly correlated (Deser 2000).

The first six LFPs all have red power spectra (Fig. 2a), with variance increasing out to multidecadal and centennial time scales. In this analysis of anomalies from the climatological seasonal cycle, the annual peak in the power spectra arises from the tendency of SST anomalies to reemerge each winter (Alexander et al. 1999). While the multimodel mean power spectra do not show distinct spectral peaks at interannual and longer time scales, this does not rule out the possibility of spectral peaks in individual models.

\section{a. Impacts on GMST}

We assess the impacts of the slow modes of variability on GMST by computing the lead-lag regression of GMST anomalies onto each LFC (center column of Fig. 1). Note that the time evolution of the GMST anomalies results in part from changes in the SST anomaly pattern over time. The modes of high-latitude warming represented by LFP-1 and LFP-2 have large and longlived impacts on GMST that are $0.056^{\circ}$ and $0.041^{\circ} \mathrm{C}$ per standard deviation at their maxima, respectively. The persistence of these patterns is such that a one-standard-deviation anomaly in the respective indices is associated with a $0.047^{\circ}$ and $0.029^{\circ} \mathrm{C}$ anomaly in decadal running-mean GMST (black lines in the center column of Fig. 1). For reference, the standard deviation of decadal-running-mean GMST anomalies in the composite of the 35 CMIP6 piControl simulations is $0.077^{\circ} \mathrm{C}$. These two modes of low-frequency variability therefore explain $\sim 37 \%$ and $\sim 14 \%$ of the variance in decadal-running-mean GMST, respectively. The impact of these modes on GMST is also evident in their coherence with GMST out to centennial time scales (Fig. 2b). 
The large impacts of LFP-1 and LFP-2 on GMST evident in the multimodel composites (Fig. 1) result in part from the large amplitudes of these modes in a few models. LFP-1 is particularly active in EC-Earth3, CNRM-ESM2.1, CNRM-CM6.1, and IPSL-CM6ALR (Fig. 3), all models that use the Nucleus for European Modeling of the Ocean (NEMO) ocean model. LFP-2 is particularly active in BCC-CSM2-MR, GFDL-ESM4, IPSL-CM6A-LR, EC-Earth3, BCC-ESM1, and GFDL-CM4 (Fig. 3), all models that use versions of either the Geophysical Fluid Dynamics Laboratory's Modular Ocean Model (MOM) or NEMO. These models are all among the CMIP6 models with the most interdecadal GMST variability (Parsons et al. 2020). However, even models that are not among these outliers show impacts of LFC-1 and LFC-2 on decadal GMST variability of $\sim 0.03^{\circ}$ and $\sim 0.018^{\circ} \mathrm{C}$ per standard deviation, respectively (as shown in section 5), and the same modes are found when these outlier models are excluded (not shown).

The impact of the PDV-like LFP-3 on GMST has a more complex temporal evolution, with its peak impact on GMST of $0.018^{\circ} \mathrm{C}$ occurring in the year before the LFC-3 maximum followed by a quick transition to a decade of anomalously cold temperatures (Fig. 1). Compared to LFP-1 and LFP-2, the amplitude of LFP-3 is relatively consistent across models (Fig. 3). However, the sign of LFP-3's lag-0 impact on decadal-mean GMST is not robust across models (as shown in section 5), a consequence of the opposite-signed impacts at lead and lag times. LFP-3 does have a robust negative impact on the rate of GMST change over the course of a decade (as shown in section 5), which is consistent with other studies that have identified the PDV as playing a role in decades of reduced GMST rise (i.e., hiatus decades; Meehl et al. 2011, 2013; Kosaka and Xie 2013; Trenberth and Fasullo 2013; England et al. 2014; Liu et al. 2016; Middlemas and Clement 2016). This could partially result from the tendency of positive PDV anomalies to follow El Niño events, which have a large impact on GMST (Pan and Oort 1983; Wigley 2000; Trenberth et al. 2002) and influence the North Pacific through the atmospheric bridge (Alexander et al. 2002). The coherence of LFC-3 and GMST is largest at 4-20-yr time scales (Fig. 2b). The impact of LFC-5' on GMST is actually somewhat larger than LFC-3 at 4-20-yr and longer time scales (Fig. 2b). LFC-5' has a peak impact on GMST of $0.036^{\circ} \mathrm{C}$ that occurs contemporaneously with the maximum in the SST pattern (i.e., at lag 0; Fig. 1).

Due to their confinement to high latitudes and their cancelling positive and negative temperature anomalies in different regions, LFP-4' and LFP-6 ${ }^{\prime}$ have relatively small impacts on GMST (Fig. 1), even at multidecadal time scales (Fig. 2b).

\section{b. Impacts on GMTOA}

Internal variability in GMTOA has much less power at decadal and longer time scales than does internal variability in GMST; while $25 \%$ of internal GMST variability is on decadal and longer time scales, only $1 \%$ of internal GMTOA variability is on these time scales (Fig. 4a). Variability in GMTOA is essentially white (i.e., its power spectral density does not vary with time scale) except for peaks in the ENSO band (3-5 yr) and at annual time scales. Variability in TOA radiation is highly coherent with variability in the net surface heat flux on decadal and longer time scales (Fig. 4b), such that the GMTOA anomaly is approximately the rate of global ocean heat uptake (cf. Palmer et al. 2011). The squared coherence between GMST and GMTOA
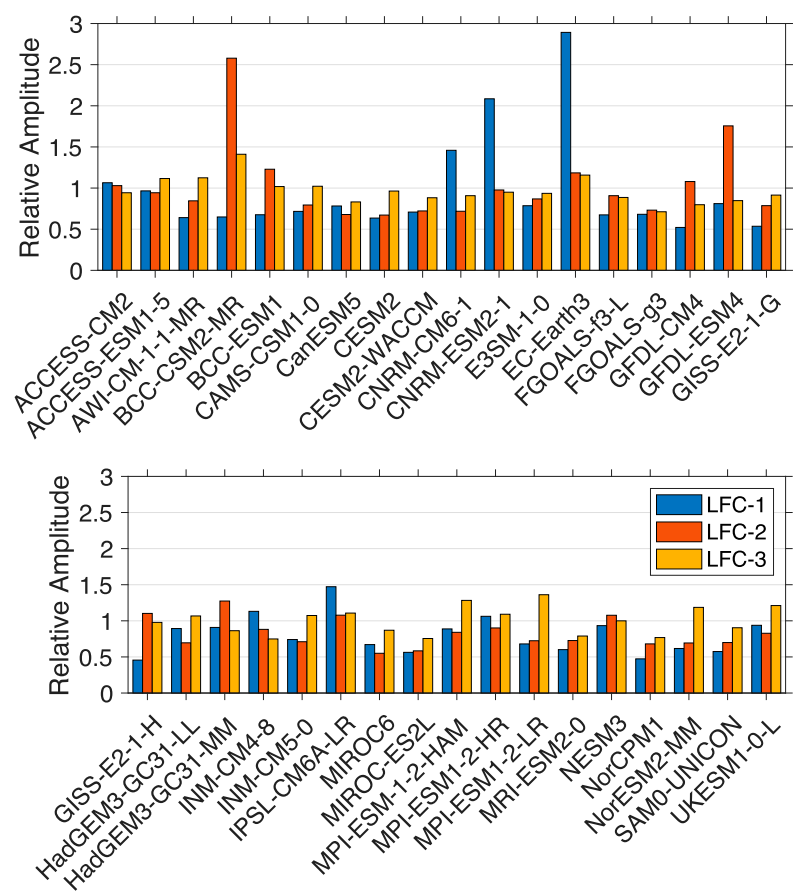

FIG. 3. Amplitude (standard deviation) of the three leading lowfrequency components (LFCs) in each of the 35 CMIP6 piControl simulations, normalized by their amplitude in the multimodel composite.

is less than 0.4 at all time scales greater than a decade (Fig. 4b), suggesting that what TOA radiation variability there is at long time scales is not determined by surface temperatures (cf. Lutsko and Takahashi 2018). This also suggests that the mechanisms controlling GMST at decadal and longer time scales do not have a big influence on GMTOA at these time scales.

We assess the impacts of the slow modes of variability on GMTOA by computing the lead-lag regression of GMTOA anomalies onto each LFC (right column of Fig. 1). Note that the time evolution of the TOA anomalies results in part from changes in the SST anomaly pattern over time. LFP-1 has a persistent TOA radiative impact of $-0.011 \mathrm{~W} \mathrm{~m}^{-2}$ in the decade following its maximum (Table 1), indicating a loss of energy from the Earth system following the warm phase of LFP-1. This GMTOA anomaly is small compared to the standard deviation of decadal-running-mean GMTOA anomalies $\left(0.070 \mathrm{~W} \mathrm{~m}^{-2}\right)$, such that LFP-1 accounts for less than $3 \%$ of the variance in GMTOA at decadal and longer time scales. The squared coherence between LFC-1 and GMTOA anomalies does not exceed 0.2 at any time scale (Fig. 2c). Thus, while LFP-1 is a major driver of decadal variability in GMST, it has a minimal impact on GMTOA.

The amplitude of global radiative anomalies associated with LFP-2 is similarly small (Fig. 1; Table 1). In addition to the weak but long-lived loss of energy after the warm phase of LFP-2, there is a net input of energy to the Earth system in the decades prior. This is evidence that LFP-2 is partially driven by TOA radiative anomalies. In section 4 , we discuss the particular radiative 


\section{a}
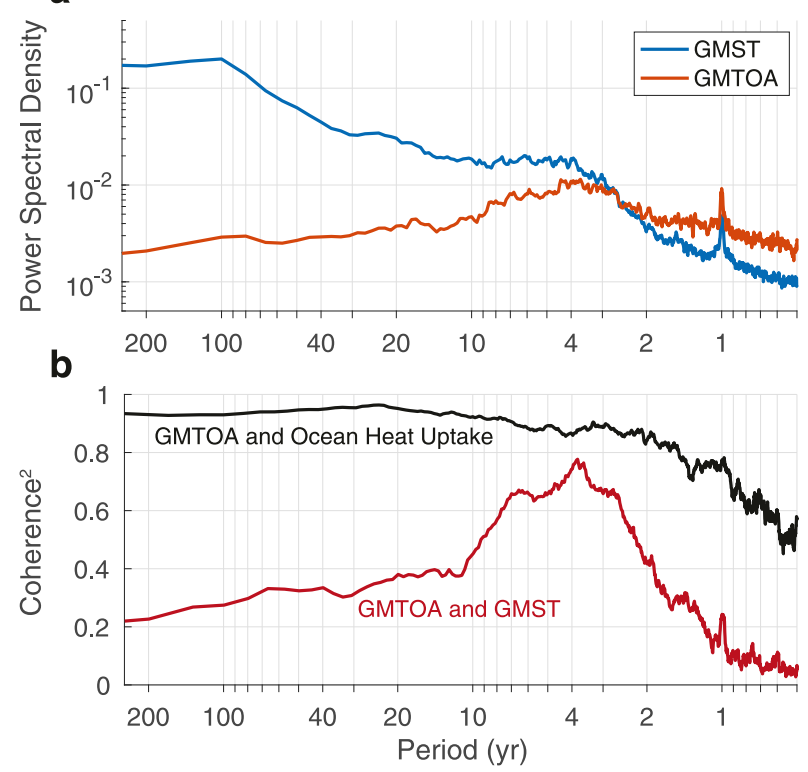

FIG. 4. (a) Power spectral density of GMST and GMTOA. (b) Squared coherence between GMST and GMTOA and between GMTOA and the global-mean net surface heat flux (ocean heat uptake). All calculations are averaged over 35 CMIP6 piControl simulations.

anomalies responsible, which point to a large role of the sea icealbedo feedback in the multidecadal variability of Southern Ocean temperature. LFC-2 has modest coherence with GMTOA anomalies at 20-50-yr time scales (Fig. 2c), but with GMTOA lagging by $\sim 90^{\circ}$ (or, equivalently, - GMTOA leading by $\sim 90^{\circ}$ ).

The warm phase of LFP-3 is associated with a short-lived negative global radiative response (energy out of the Earth system) followed by a persistent cooling of GMST and an associated positive global radiative response (energy into the Earth system; Fig. 1). Despite the shorter time scale of LFC-3 compared to LFC1 and LFC-2, the associated anomalies in decadal running-mean GMTOA are nearly as large. LFP-5' ${ }^{\prime}$ has a similar magnitude of impact on GMTOA to LFP-3, but with a different time evolution. Like LFP-2, LFP-5' appears to be amplified by global radiative feedbacks (i.e., during the evolution of an LFP-5' warm event, TOA radiative fluxes are warming the surface at lead times and cooling the surface at lag times). LFP-4' leads to TOA energy loss in the $\sim 5$ years following its maximum. LFP- $6^{\prime}$ has a minimal impact on GMTOA. One overall takeaway is that the time evolution of global radiative feedbacks varies widely across different types of low-frequency variability.

\section{c. Impact of ENSO on global climate at decadal time scales}

The dominant mode of interannual variability in the climate system is ENSO, which is often characterized by the Niño-3.4 index (SST anomalies averaged over $5^{\circ} \mathrm{S}-5^{\circ} \mathrm{N}, 120^{\circ}-170^{\circ} \mathrm{W}$ ). The broader SST anomaly pattern associated with Niño-3.4, based on a regression of local SST anomalies on the Niño-3.4 index, is shown in Fig. 5. In the models studied, Niño-3.4 variance peaks at 3-5-yr time scales (Fig. 2a). Here, we are interested in characterizing the impact of ENSO on global climate at decadal and longer time scales.

Because Niño-3.4 can be correlated with the leading LFCs (e.g., it has a correlation of 0.12 with LFC-3), we also consider the representation of ENSO within the LFCA. Although ENSO has power at decadal and longer time scales (Fig. 2a), its large amplitude at interannual time scales gives it a low ratio of interdecadal-to-intradecadal variance. For this reason, much of the ENSO-like variance is captured by the least-low-frequency component (LLFC; Fig. 5), which is the linear combination of the included EOFs with the minimum ratio of interdecadal-tointradecadal variance. The correlation between the LLFC and Niño-3.4 is 0.77. Their spatial patterns are similar in the equatorial Pacific (within $\pm 10^{\circ}$ of the equator; Fig. 5), but the LLFC does not include associated SST anomalies in the extratropical North and South Pacific and in the Indian Ocean. Similar to Niño-3.4, the LLFC has its peak amplitude at 3-4-yr time scales; however, it has a factor of 2 less variance than ENSO at decadal and longer time scales (Fig. 2a). This shows that the LLFC isolates the interannual component of ENSO. The LFC with the next-highest correlation with the Niño-3.4 index is LFC-60 (i.e., the fifth LLFC), with a correlation of 0.32. LFC-60 shows warming in the central equatorial Pacific, cooling in the KuroshioOyashio Extension, and a positive anomaly in the Indian Ocean dipole (IOD) (not shown), as is characteristic of central Pacific El Niño events (Takahashi et al. 2011; Capotondi et al. 2015; Timmermann et al. 2018). Together these two LLFCs account for $69 \%$ of the variance in Niño-3.4.

ENSO, as captured by Niño-3.4, has a large impact on GMST (Pan and Oort 1983; Wigley 2000; Trenberth et al. 2002). In the CMIP6 models, the maximum GMST anomaly of $0.08^{\circ} \mathrm{C}$ per standard deviation occurs $2-3$ months after the maximum Niño-3.4 anomaly (Fig. 5). There are also weaker

TABLE 1. Multimodel median statistics of decadal GMST and GMTOA variability. The stdev. column gives the standard deviation of each quantity. The LFC-1, LFC-2, LFC-3, LLFC, and Niño-3.4 columns give the lag-5 covariance of the corresponding quantity with each of these indices (i.e., the anomaly in the decade following the maximum in each index, in units of the corresponding quantity per standard deviation). The global climate feedback is calculated as the 10-yr running-mean GMTOA anomaly divided by the 10-yr running-mean GMST anomaly. The lag-5 covariance is used because of intermodel differences in the sign of some GMST and GMTOA anomalies at lag 0 (see section 5).

\begin{tabular}{|c|c|c|c|c|c|c|}
\hline & Stdev. & LFC-1 & LFC-2 & LFC-3 & LLFC & Niño-3.4 \\
\hline 10-yr running-mean GMST $\left({ }^{\circ} \mathrm{C}\right)$ & 0.063 & 0.021 & 0.016 & -0.005 & 0.003 & 0.005 \\
\hline 10-yr running-mean GMTOA $\left(\mathrm{W} \mathrm{m}^{-2}\right)$ & 0.064 & -0.011 & -0.009 & 0.004 & -0.010 & -0.017 \\
\hline Global climate feedback $\left(\mathrm{W} \mathrm{m}^{-2} \mathrm{~K}^{-1}\right)$ & -1.03 & -0.50 & -0.57 & -0.70 & -2.94 & -3.20 \\
\hline
\end{tabular}



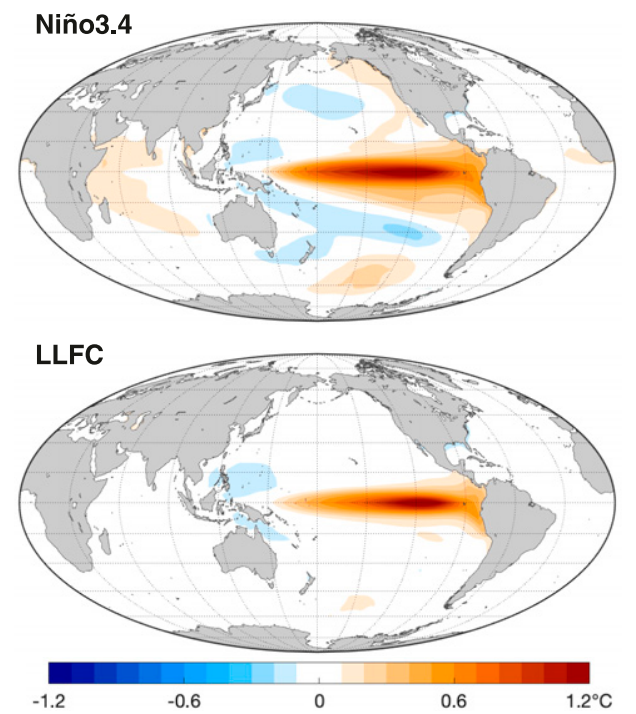
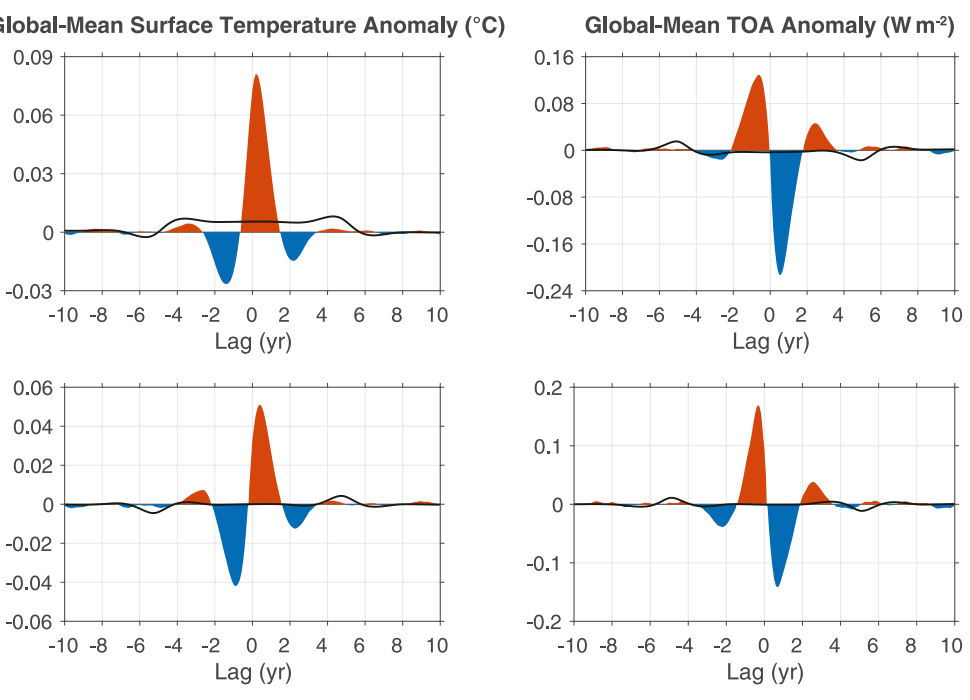

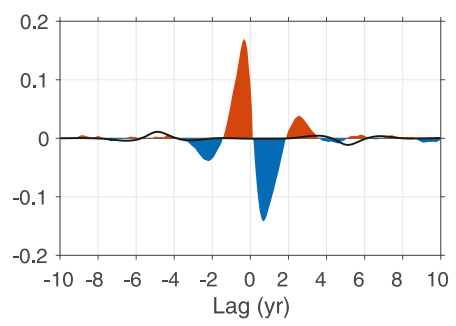

FIG. 5. ENSO and its impacts on GMST and GMTOA, as characterized by (top) Niño-3.4 and (bottom) the least low-frequency component (LLFC). (left) The SST anomaly pattern per standard deviation in the associated index. (center) The lead-lag regression of monthly GMST anomalies on the associated normalized ENSO index. (right) The lead-lag regression of monthly GMTOA anomalies on the associated normalized ENSO index. Black lines show the same lead-lag regressions, but for the 10-yr running means of GMST and GMTOA. Positive lags indicate anomalies that occur after the maximum anomaly in the ENSO index. All calculations are averaged over 35 CMIP6 models.

negative GMST anomalies at $\pm \sim 2$ years, which are associated with the structure of the ENSO autocorrelation, because there is a tendency for La Niña years to follow El Niño years. The positive GMST anomaly associated with the LLFC is notably muted compared to that associated with Niño-3.4; it peaks at $0.05^{\circ} \mathrm{C}$ per standard deviation at a lag of 5-6 months (Fig. 5). The LLFC leads Niño-3.4 by 1-2 months, which explains the greater GMST lag.

The coherence with GMST as a function of time scale is markedly different between Niño-3.4 and the LLFC (Fig. 2b); while they have similarly large coherence with GMST in the ENSO band (2-5yr), the coherence between the LLFC and GMST drops off quickly at longer time scales, such that there is no significant coherence between the LLFC and GMST at $30-y r$ and longer time scales. In contrast, Niño-3.4 still has a squared coherence of $\sim 30 \%$ with GMST at time scale ranging from multidecadal to centennial, although Niño-3.4 variance is small at these time scales. This analysis suggests that some of the impact of ENSO on GMST, especially that at decadal and longer time scales, comes from its teleconnections to regions outside the equatorial Pacific (which are not emphasized in the LLFC).

The impact of ENSO on GMTOA is asymmetric about lag 0 (Fig. 5), meaning that the energy content of the Earth system is maximum near the peak of El Niño events, a result that has been confirmed in observations (Johnson and Birnbaum 2017). Peak energy input into the Earth system is $0.13 \mathrm{~W} \mathrm{~m}^{-2}$ per standard deviation 7 months before Niño-3.4 maxima and the peak energy loss is $0.21 \mathrm{~W} \mathrm{~m}^{-2}$ per standard deviation 6 months after Niño-3.4 maxima. The lead-lag covariance between GMTOA and ENSO is similar when quantified based on the LLFC, with modest changes in the peak radiative anomalies and a $\sim 2$-month shift in the timing. Niño-3.4 and the LLFC explain a similarly large fraction of GMTOA variance in the ENSO band (3-5yr), but the LLFC explains significantly less at longer time scales (Fig. 2c).

While the LLFC captures many of the same features of ENSO's impact on global climate as Niño-3.4, it removes most of its variance and associated impacts on decadal and longer time scales. The LLFC removes some of the asymmetry between El Niño and La Niña events (not shown), and it may therefore underestimate the influence of extreme El Niños on global climate. On the other hand, Niño-3.4 is not independent of LFC-3, especially on time scales between 7 and 40 years, where their squared coherence (shared variance) is 0.3 . These caveats should be kept in mind when using either index. To include the full range of ENSO impacts on global climate, we will focus on the more widely used Niño-3.4 index for the analysis in the following sections.

\section{d. Comparison of global climate feedbacks}

We have shown that the leading modes of low-frequency variability have a large impact on GMST but a small impact on GMTOA (Figs. 1 and 2b,c). To quantify the global radiative feedback associated with these modes and compare with the global radiative feedback associated with ENSO, we focus on the global climate anomalies in the decade following the maximum in each index (i.e., the 5-yr lagged covariance between 10-yr running mean GMST or GMTOA and each index; Table 1). This approach avoids near zero decadal-mean GMST anomalies associated with LFC-3 and the LLFC at lag 0 (Figs. 1 and 5). The ratio of the GMTOA anomaly and the GMST anomaly quantifies a global climate feedback associated with each mode of variability in the decade following its maximum [i.e., considering one mode at a time in Eq. (5)]. GMTOA anomalies are defined as negative for energy loss from the Earth system, by convention, such that negative values of the global 
climate feedback quantify the strength of the negative (damping) feedback. The global climate feedbacks for LFC-1 and LFC-2 are small: -0.50 and $-0.57 \mathrm{~W} \mathrm{~m}^{-2} \mathrm{~K}^{-1}$, respectively (Table 1 ). For comparison, the global climate feedback associated with an ECS of $3.5^{\circ} \mathrm{C}$ is approximately $-1.1 \mathrm{~W} \mathrm{~m}^{-2} \mathrm{~K}^{-1}$. If the global climate feedback was $-0.5 \mathrm{~W} \mathrm{~m}^{-2} \mathrm{~K}^{-1}$ for a warming in response to greenhouse gas emissions, this would correspond to an ECS of $8^{\circ} \mathrm{C}$ [Eq. (2)]. The global climate feedback associated with the cooling following a warm phase of LFC-3 is only slightly stronger: $-0.70 \mathrm{~W} \mathrm{~m}^{-2} \mathrm{~K}^{-1}$.

In contrast, ENSO has a small impact on decadal-mean GMST but a large impact on decadal-mean GMTOA (Table 1). This results in a large global climate feedback associated with the decay phase of ENSO: -2.94 or $-3.20 \mathrm{~W} \mathrm{~m}^{-2} \mathrm{~K}^{-1}$ when quantified by the LLFC or Niño-3.4, respectively. Radiative feedbacks this large for a warming in response to greenhouse gas emissions would correspond to an ECS of $1.25^{\circ} \mathrm{C}$. In section 5, we consider how the contrasting global radiative feedback strengths associated with the LFCs and ENSO lead to differences in their effects on estimates of climate sensitivity from short records.

\section{Mechanisms of global radiative feedback}

A key conclusion of our analysis thus far is that LFC-1 and LFC-2 have a weak global radiative feedback, such that their large impact on GMST on decadal and longer time scales does not translate into a large impact on GMTOA. In this section, we examine the particular global energy budget changes that result in LFC-1 and LFC-2 having a weak global radiative feedback and compare to the global energy budget changes associated with LFC-3 and Niño-3.4. We focus on changes during the positive phases of these modes of variability, but the linearity of our analysis implies that the same conclusions also apply, with opposite sign, to the negative phases of these modes of variability.

\section{a. Atlantic multidecadal variability}

Atlantic multidecadal variability (AMV), as characterized by LFC-1, has a small GMTOA anomaly due to the net result of large cancelling anomalies in the longwave (LW) and shortwave (SW) components (Fig. 6a). In the warm phase of the AMV, there is anomalous global energy loss resulting from a strengthening of outgoing longwave radiation. This negative feedback acts to damp the warm temperature anomalies. LW anomalies are largest in the high latitudes of the Northern Hemisphere (Fig. 6b), where surface air temperature anomalies are largest. There are also interesting spatial features such as the presence of positive LW anomalies in the Northern Hemisphere tropics and negative LW anomalies in the Southern Hemisphere tropics, which indicates a northward shift of the intertropical convergence zone (ITCZ) in response to warming of the high latitudes of the Northern Hemisphere (Chiang and Bitz 2005; Broccoli et al. 2006). The LW component alone would amount to a large global radiative feedback $\left(-1.5 \mathrm{~W} \mathrm{~m}^{-2} \mathrm{~K}^{-1}\right.$ based on decadal anomalies centered at lag $0)$. However, in the global mean, these LW anomalies are mostly cancelled by anomalies in SW.
SW anomalies are positive (net energy gain) in most regions of the globe (Fig. 6d). They are largest in the North Atlantic, but there are also anomalies in the North Pacific and Arctic. The SW anomalies are particularly large near the sea ice edge, where warm SST anomalies lead to sea ice loss and invoke a positive sea ice-albedo feedback. SW anomalies in the midlatitude northeast Atlantic are partially associated with a reduction in low-cloud cover, as evident in the reduction in reflected shortwave radiation. This supports the idea that cloud feedbacks act to amplify the AMV (Brown et al. 2016; Yuan et al. 2016; Bellomo et al. 2016). The positive cloud and sea ice-albedo feedbacks act to cancel the negative OLR feedback such that the net feedback is weakly negative $\left(-0.22 \mathrm{~W} \mathrm{~m}^{-2} \mathrm{~K}^{-1}\right.$, based on decadal anomalies centered at lag 0 ).

The net loss of energy at TOA comes almost entirely from the ocean, with little change in the energy stored in the global atmosphere (Fig. 6a). The ocean heat loss at the surface is concentrated in the subpolar North Atlantic (Fig. 6c), where the positive SSTs anomalies are largest (Fig. 1). This indicates the dominant role of ocean heat transport convergence anomalies [and thus the role of ocean circulations such as the Atlantic meridional overturning circulation (AMOC)] in sustaining decadal SST anomalies in the North Atlantic (Wills et al. 2019a and references therein).

\section{b. Southern Ocean multidecadal variability}

The lead-lag regression of GMTOA on LFC-2 [Southern Ocean multidecadal variability (SOMV)] shows that the Earth system is gaining energy in the decades before and losing energy in the decades after the peak of a warm phase of the SOMV (Fig. 7a). However, the SW and LW components each remain the same sign throughout the decades surrounding a SOMV event. The LW anomaly during an SOMV warm event is negative (a positive anomaly in outgoing longwave radiation) and is concentrated in the high latitudes of the Southern Hemisphere (Fig. 7b), where surface air temperature anomalies are largest. The LW anomalies also show a hint of a southward ITCZ shift (positive anomalies in the Southern Hemisphere tropics and negative anomalies in the Northern Hemisphere tropics).

The global LW anomaly would strongly damp GMST anomalies associated with SOMV $\left(-1.6 \mathrm{~W} \mathrm{~m}^{-2} \mathrm{~K}^{-1}\right.$ based on decadal anomalies centered at lag 0 ) if it were not for a compensating positive anomaly in SW. The SW anomaly is concentrated in regions where sea ice is lost due to warming of the Southern Ocean (Fig. 7d), suggesting that it is dominated by the sea ice-albedo feedback, although we find that cloud radiative changes also play a role (not shown). As was the case for AMV, the shortwave feedbacks associated with SOMV act to cancel the negative LW feedback such that the net feedback is weakly positive $\left(0.16 \mathrm{~W} \mathrm{~m}^{-2} \mathrm{~K}^{-1}\right.$, based on decadal anomalies centered at lag 0$)$.

The GMTOA changes are balanced by changes in ocean heat content, with little contribution from atmospheric energy storage (Fig. 7a). The ocean heat loss and heat gain occur in different regions (Fig. 7c), with ocean heat loss occurring along the coast of Antarctica and ocean heat uptake occurring farther north, in the Indian and Pacific sectors of the Southern Ocean. 

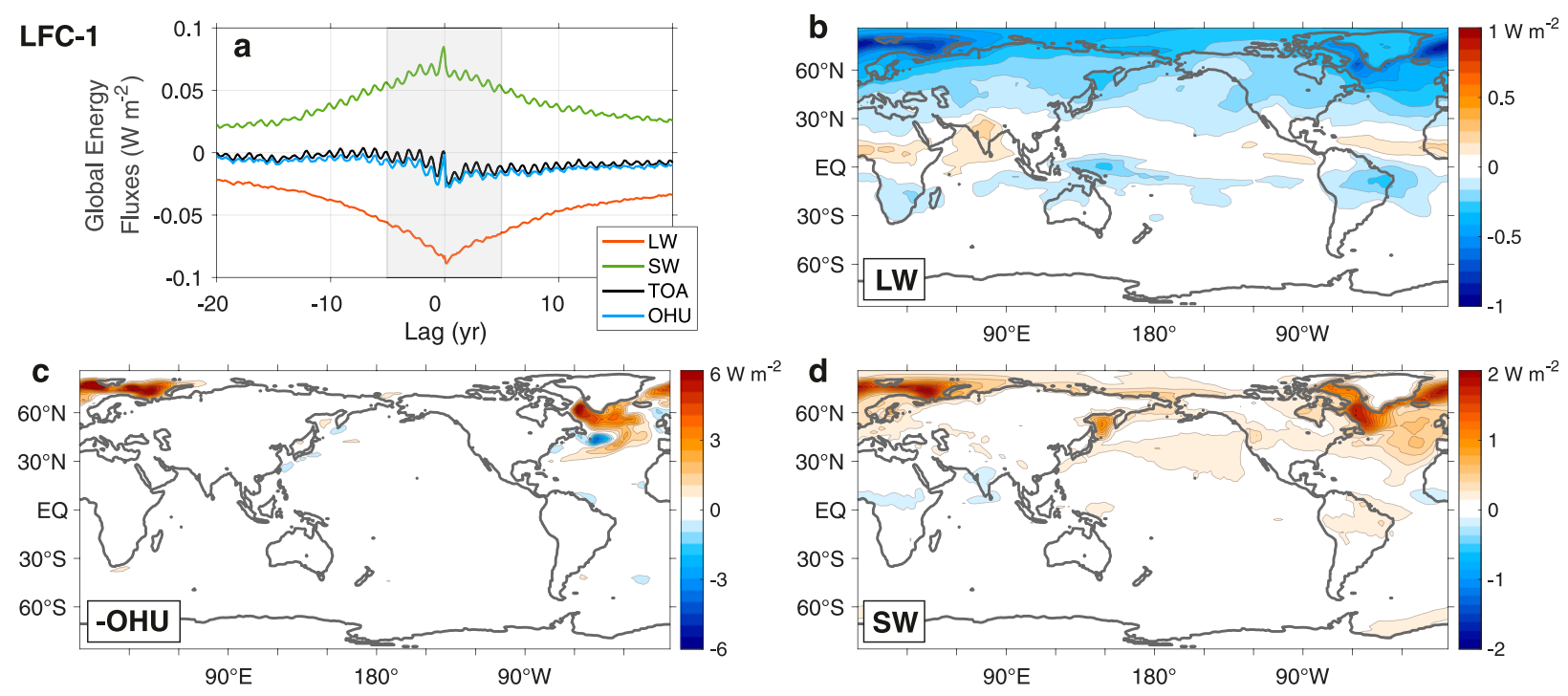

FIG. 6. Decadal-mean impact of the AMV-like LFC-1 on the global energy budget. (a) Lead-lag regression of net incoming longwave radiation at TOA $(\mathrm{LW})$, net incoming shortwave radiation at TOA (SW), net incoming radiation at TOA, and net ocean heat uptake (OHU) on LFC-1. Positive TOA flux anomalies correspond to a net energy input to the Earth system. Lag times indicate anomalies that lag LFC-1. Also shown are maps of 10-yr running-mean anomalies in (b) LW, (c) -OHU, and (d) SW associated with a one-standarddeviation anomaly in LFC-1. The gray shading in (a) shows the averaging period used in the other subpanels. All calculations are averaged over 35 CMIP6 models.

The decadal ocean heat loss anomalies around Antarctica, in regions where SST anomalies are positive (Fig. 1), indicate a role of ocean heat transport convergence in sustaining these anomalies. Studies of models with large amplitude SOMV (most of which use either the NEMO or MOM ocean models) have generally found this variability to be linked to open-ocean deep convection within polynyas (Martin et al. 2013; Pedro et al. 2016; Behrens et al. 2016; Reintges et al. 2017; Zhang et al. 2017; Cabré et al. 2017), which does not occur in all CMIP6 models. However, the presence of this variability in all of the CMIP6 models studied (albeit with a large spread in amplitudes and time scales) suggests that this mode of variability may be more general.

\section{c. Pacific decadal variability}

Pacific decadal variability (PDV), as characterized by LFC3 , has a more complicated lead-lag relationship with LW and SW anomalies than the first two LFCs (Fig. 8a). Global-mean LW anomalies are negative in the decade leading up to the PDV warm phase (when temperatures are warm in the Gulf of Alaska and cold in the Kuroshio-Oyashio Extension), before switching signs 1.5 years after the PDV maximum. This time evolution is similar but opposite in sign to PDV GMST anomalies (Fig. 1), such that LW anomalies act to damp GMST anomalies. LW anomalies in the decade surrounding a PDV maximum are dominated by positive anomalies (e.g., from more high cloud cover) in the central and western equatorial Pacific $\left(135^{\circ} \mathrm{E}-165^{\circ} \mathrm{W}\right)$ and negative anomalies (e.g., from less high cloud cover) in the warm pool, the South Pacific convergence zone, and the northeastern subtropical Pacific (Fig. 8b). SW anomalies are opposite in sign in each of these regions (Fig. 8d). The SW anomalies in the North Pacific more closely resemble anomalies in SST than do LW anomalies. These North Pacific SW anomalies are consistent with a reduction in low cloud cover in regions of positive SST anomalies, as has been observed (Schmeisser et al. 2020). This positive feedback, particularly in the eastern Pacific, has been suggested to play a role in the amplitude and persistence of the PDV (Bellomo et al. 2014).

In the global mean, positive SW anomalies cancel with negative LW anomalies in the years leading up to a PDV warm event (Fig. 8a). The large negative GMTOA anomaly near lag 0 is associated with changes toward more negative values in both the SW and LW components. This negative GMTOA anomaly helps to switch the sign of GMST anomalies. SW anomalies remain negative (a positive feedback on negative GMST anomalies) during the decade after a PDV event, but they are smaller in magnitude than the positive LW anomalies. The net effect of the SW and LW terms is near zero until a year before a PDV event, followed by net energy loss in the $\sim 3$-yr period surrounding a PDV event and then net energy gain in the following $\sim 8$ years of negative GMST anomalies (Fig. 8a).

As with other modes of low-frequency variability, the GMTOA changes are balanced by changes in ocean heat content. Ocean heat loss during the peak of the PDV event occurs primarily in the Kuroshio and the central and western equatorial Pacific $\left(135^{\circ} \mathrm{E}-165^{\circ} \mathrm{W}\right)$; ocean heat uptake in the following decade occurs primarily in the Oyashio and the same region of the central and western equatorial Pacific. Averaged over 10 years, this amounts to a northward transport of heat from the Kuroshio into the Oyashio (Fig. 8c). This anomalous ocean heat transport occurs through an adjustment of the 

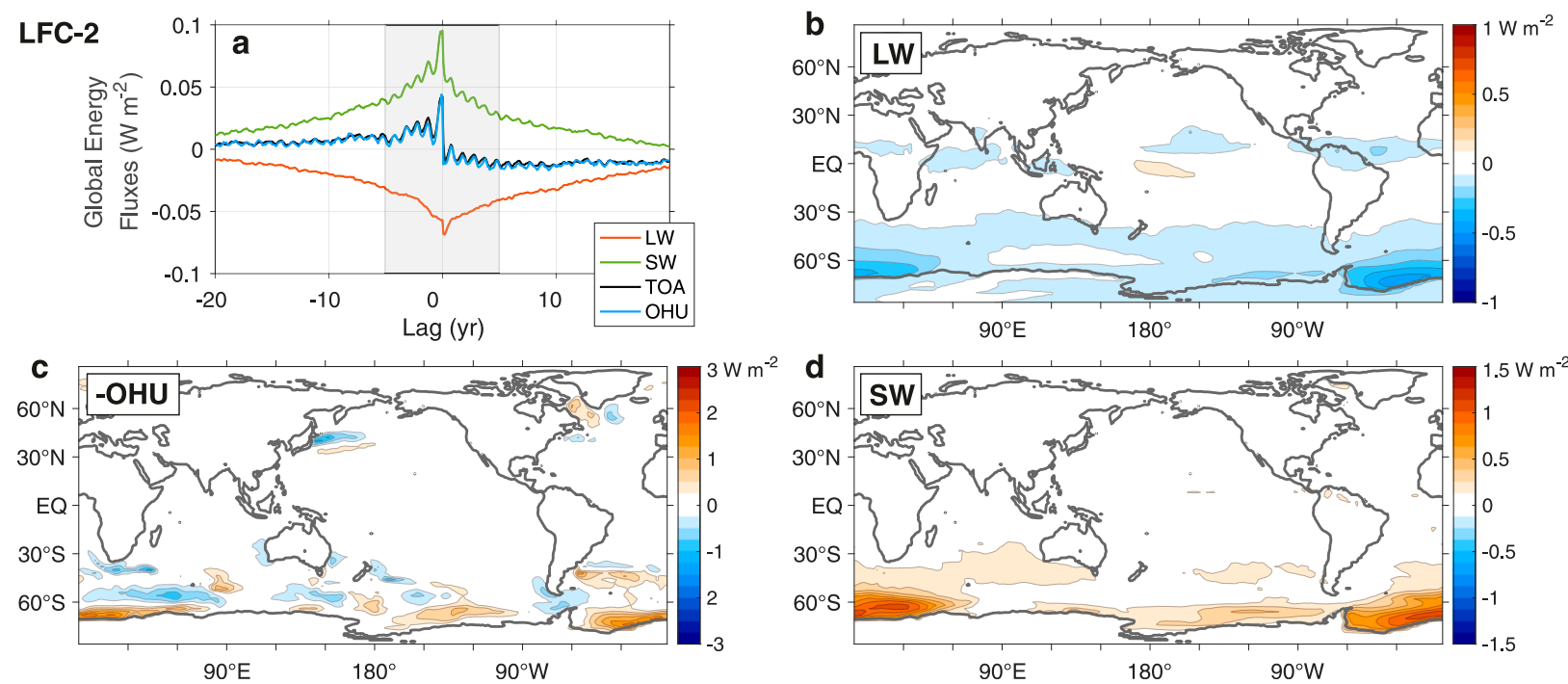

FIG. 7. Decadal-mean impact of LFC-2 (Southern Ocean multidecadal variability) on the global energy budget. Panels are as described in Fig. 6.

ocean gyre circulation to changes in wind forcing (Wills et al. $2019 b$ and references therein).

\section{d. El Niño-Southern Oscillation}

The global energy budget changes associated with Niño-3.4 are opposite in sign between lead times and lag times (Fig. 9a) and largely cancel out in a decadal average centered about lag 0 (Fig. 12a). We therefore focus on global energy budget anomalies separately in the year preceding and the year following an ENSO event (i.e., the build up and decay phase of an ENSO event). Note that our regression approach weights El Niño and La Niña events equally. The lead-lag structure of TOA is similar between El Niño and La Niña events (inset in Fig. 9a), but with the build up phase being weaker and slower and the decay phase being stronger and faster for El Niño events compared to La Niña events.

The lead-lag structure of GMTOA (Fig. 9a) is the net result of a LW component, which evolves in tandem to and acts to damp GMST anomalies (cf. Fig. 1), and a SW component, which evolves in quadrature with GMST anomalies (Lutsko and Takahashi 2018). For the months around a peak ENSO event, LW and SW anomalies largely cancel, leading to small anomalies in GMTOA. At lead or lag times larger than a few months, LW and SW anomalies reinforce each other, leading to large anomalies in GMTOA.

At both lead and lag times, LW and SW anomalies are largest in the central and western equatorial Pacific (Figs. 9d,f), where they show the eastward shift of deep convection
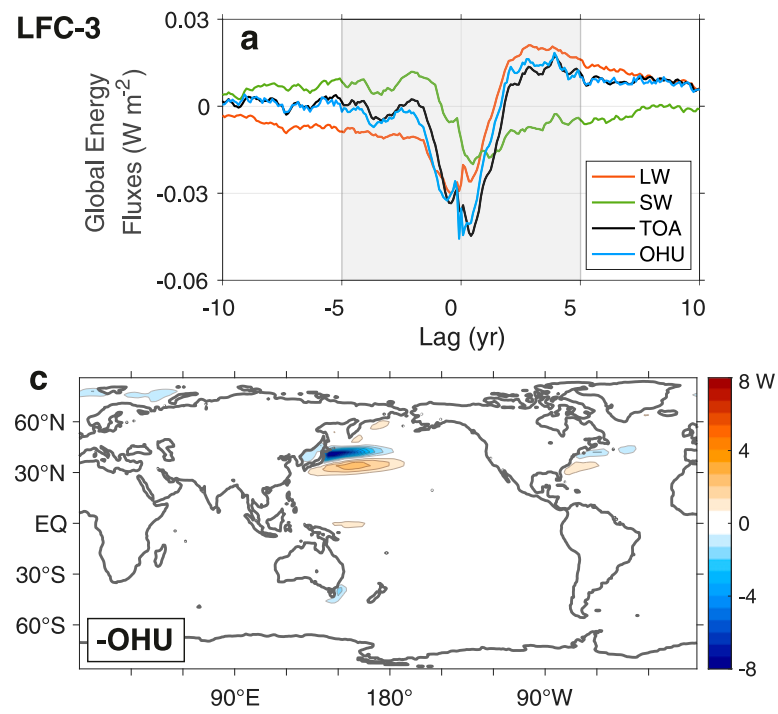
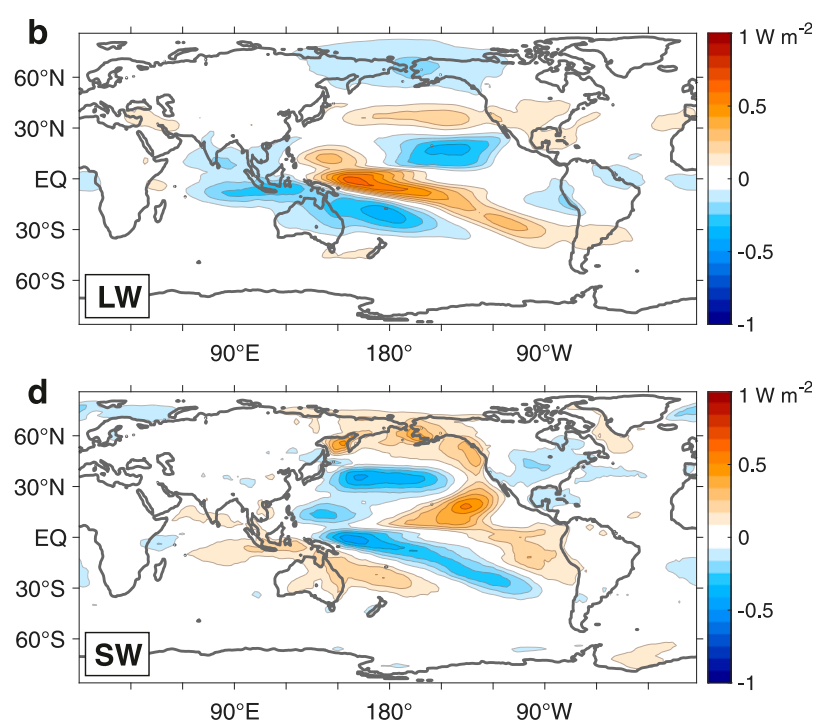

FIG. 8. Decadal-mean impact of the PDV-like LFC-3 on the global energy budget. Panels are as described in Fig. 6. 

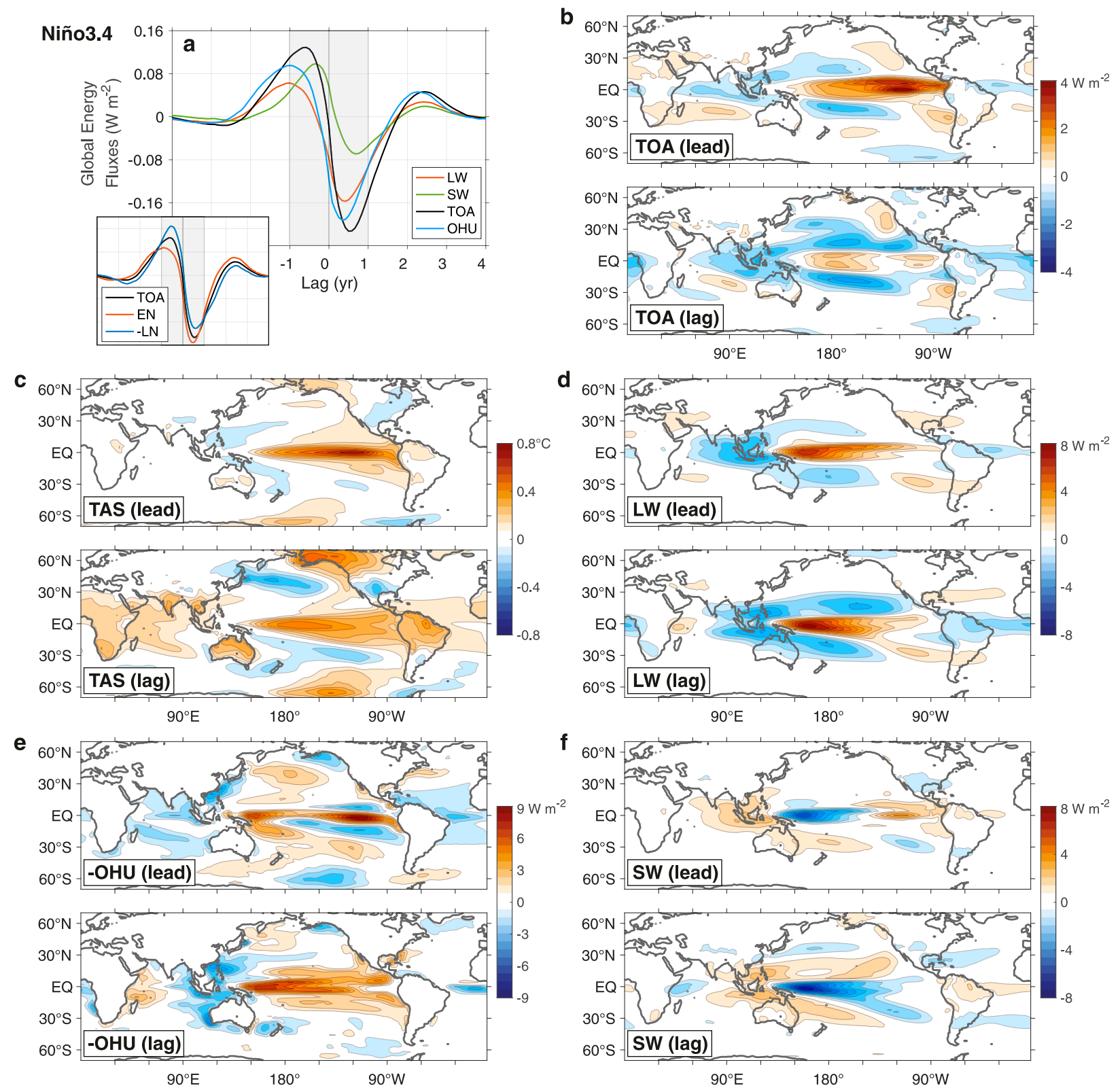

FIG. 9. Impact of ENSO on the global energy budget. (a) Lead-lag regression of net incoming longwave radiation at TOA (LW), net incoming shortwave radiation at TOA (SW), net incoming radiation at TOA, and net ocean heat uptake (OHU) on Niño-3.4. Positive TOA flux anomalies correspond to a net energy input to the Earth system. Lag times indicate anomalies that lag Niño-3.4. The inset shows the leadlag regression of TOA separately for El Niño (EN) and La Niña (LN), normalized such that their lead-lag regressions have the same variance across all lead and lag times. Also shown are maps of anomalies in (b) net incoming radiation at TOA, (c) surface air temperature (TAS), (d) LW, (e) - OHU, and (e) SW associated with a one-standard-deviation anomaly in Niño-3.4. Top subpanels show anomalies in the year before the Niño-3.4 maximum; bottom subpanels show anomalies in the year after (i.e., 1-yr running mean anomalies at a lead time of 6 months and a lag time of 6 months, respectively). The gray shading in (a) shows the averaging periods used in the other subpanels. All calculations are averaged over 35 CMIP6 models.

during El Niño events (Rasmusson and Carpenter 1982; Deser and Wallace 1990). These anomalies largely cancel in the net TOA radiation (Fig. 9b). At lead times, net TOA anomalies are largest in the eastern Pacific (Fig. 9b), where the combined LW and SW feedbacks provide a positive feedback on ENSO growth (Bellomo et al. 2014; Rädel et al. 2016; Lutsko
2018; Middlemas et al. 2019). At lag times, net TOA anomalies are largest in the subtropical Pacific and the warm pool (Fig. 9b), where negative LW anomalies (positive OLR anomalies) dominate over positive SW anomalies, resulting in a net loss of energy in regions of weakly positive surface air temperature anomalies (Fig. 9c). These differences in radiative anomalies between 
lead times and lag times result in a net energy gain leading up to an El Niño event and a net energy loss afterward. The large but cancelling anomalies in lead and lag times can result in biases in estimates of climate sensitivity from short records if the averaging period does not include the full period of energy gain and loss, as we will discuss in section 5 .

Temperature and radiation anomalies in the subtropics are larger in the year after a maximum in Niño-3.4 than in the year before. Anomalies in these fields therefore cover a broader range of latitudes at lag times, when the temperature pattern is similar to the PDV (Zhang et al. 1997). Note, however, that the decadal variability captured by the PDV-like LFC-3 is much more focused in the midlatitudes (Fig. 1) and occurs due to midlatitude processes that are only loosely connected to ENSO (Wills et al. 2019b).

The evolution of global-mean ocean heat uptake (OHU) during an ENSO event looks similar to that of GMTOA (Fig. 9a), but with a slight lead such that the ocean heat content is maximum at a lead time of $\sim 3$ months (where $\mathrm{OHU}=0$ ) and the atmospheric heat content is maximum at a lag time of $\sim 4$ months (where TOA $=$ OHU). The total energy content of the Earth system peaks just before lag 0 (where TOA $=0$ ), similar to what is found in observational estimates of ocean heat content (Johnson and Birnbaum 2017). Ocean heat uptake at lead times primarily occurs in the Atlantic and Indian Oceans, off the equator in the tropical eastern Pacific, and along the coast of East Asia (Fig. 9e). Along the equator, the ocean is already losing energy in the year before an El Niño event (Fig. 9e), but this energy loss covers a much larger area in the year following an El Niño event. This heat loss from the ocean surface acts to damp the SST anomalies set up by anomalies in ocean heat transport and upwelling (Zebiak and Cane 1987; Philander 1989; Deser and Wallace 1990). Locally, the net surface heat flux anomalies are larger in magnitude than the anomalies in net TOA radiation (Fig. 9e; cf. Fig. 9b, note the different color scales). They are a dominant factor in driving anomalies in tropical overturning circulations such as the Walker circulation, partly through their influence on largescale SST and sea level pressure gradients (Chiang et al. 2001; Adames and Wallace 2017) and partly through their direct influence on the strength of ascent in deep convective regions (Wills et al. 2017).

\section{Impact of internal variability on climate sensitivity estimates}

Armed with an improved understanding of how modes of internal variability give rise to anomalies in GMST and global radiative feedbacks, we now turn to the question of how these modes of variability can impact estimates of climate sensitivity based on historical GMST and TOAradiative-imbalance anomalies. To consider the impact of internal variability on observational estimates of climate sensitivity, we split $\bar{T}_{\text {obs }}$ and $\bar{N}_{\text {obs }}$ in Eq. (3) into forced and internal components:

$$
\text { EffCS }=\frac{\bar{F}_{2 \times \mathrm{CO}_{2}}\left(\bar{T}_{\text {forced }}+\bar{T}_{\text {internal }}\right)}{\bar{F}_{\text {obs }}-\bar{N}_{\text {forced }}-\bar{N}_{\text {internal }}} .
$$
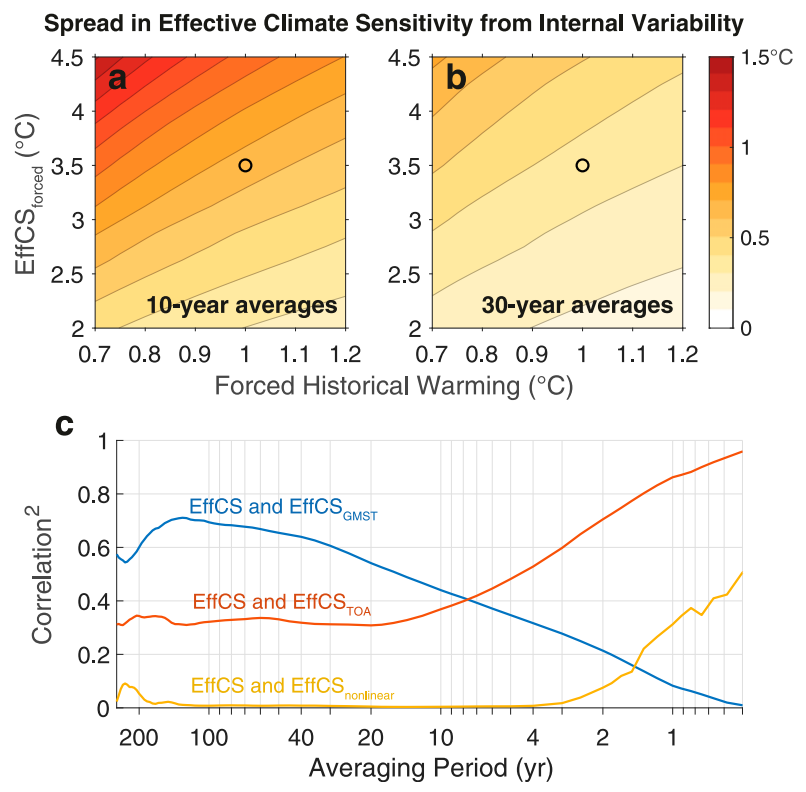

FIG. 10. Two-standard-deviation $(\approx 95 \%)$ spread in effective climate sensitivity (EffCS) from superimposing internal variability from CMIP6 piControl simulations on an assumed externally forced historical warming ( $\bar{T}_{\text {forced }} ; x$ axis) and a radiative response corresponding to a forced effective climate sensitivity (EffCS forced$y$ axis), where EffCS is calculated from (a) 10-yr running averages and (b) 30-yr running averages of Eq. (6). Values are the median across 35 CMIP6 models. Black circles show the values of $\bar{T}_{\text {forced }}$ and EffCS $_{\text {forced }}$ used in Figs. 11 and 12. (c) Multimodel mean squared correlation between anomalies in EffCS and its components due to internal variability in GMST and GMTOA, as a function of the averaging period used to compute EffCS. See the appendix for more details of this decomposition.

We take representative externally forced anomalies in GMST ( $\left.\bar{T}_{\text {forced }}\right)$ and GMTOA $\left(\bar{N}_{\text {forced }}\right)$ and superimpose internal variability in GMST ( $\left.\bar{T}_{\text {internal }}\right)$ and GMTOA $\left(\bar{N}_{\text {internal }}\right)$, as diagnosed from the CMIP6 piControl simulations.

We first compute the total spread in EffCS due to all types of internal variability by taking the standard deviation of the 10and 30-yr running-mean EffCS, based on Eq. (6) (Figs. 10a and $10 \mathrm{~b}$, respectively). The spread in EffCS depends on the amount of externally forced historical warming $\left(\bar{T}_{\text {forced }}\right)$ and the radiative response to that historical warming, quantified here with an effective climate sensitivity:

$$
\mathrm{EffCS}_{\text {forced }}=\frac{\bar{F}_{2 \times \mathrm{CO}_{2}} \bar{T}_{\text {forced }}}{\bar{F}_{\text {obs }}-\bar{N}_{\text {forced }}} .
$$

The spread in EffCS is largest for a small historical warming or a weak global radiative response (higher EffCS $_{\text {forced }}$ ). For an externally forced historical warming $\bar{T}_{\text {forced }}=1^{\circ} \mathrm{C}$ and radiative response $\bar{F}_{\text {obs }}-\bar{N}_{\text {forced }}=1.14 \mathrm{~W} \mathrm{~m}^{-2}$ (corresponding to EffCS $_{\text {forced }}=3.5^{\circ} \mathrm{C}$ ), the two-standard-deviation spread in EffCS due to internal variability would be $\pm 0.65^{\circ} \mathrm{C}$ based on 10 -yr averages or $\pm 0.36^{\circ} \mathrm{C}$ based on 30 -yr averages (circles in Figs. 10a and $10 \mathrm{~b}$, respectively). At decadal and longer time scales, internal variability primarily influences EffCS through 
its impact on GMST rather than through its influence on GMTOA (Fig. 10c; see the appendix for a full decomposition of the contributions to spread in EffCS). This reflects the red power spectrum of GMST, which shows large variability on decadal and longer time scales (Fig. 4a), whereas GMTOA has a relatively white power spectrum, with peak variance in the ENSO band.

The diagnosed spread in EffCS based on internal variability in CMIP6 models $\left( \pm 0.65^{\circ} \mathrm{C}\right.$ based on 10 -yr averages $)$ is comparable to the range of EffCS across historical simulations from the 100 -member MPI-ESM1.1 large ensemble $\left( \pm 0.95^{\circ} \mathrm{C}\right.$ based on 10-yr averages; Dessler et al. 2018). This spread in EffCS due to internal variability is smaller than the observational uncertainty range of $1.2^{\circ}-3.9^{\circ} \mathrm{C}$ given in Otto et al. (2013), which includes observational uncertainty in GMST, radiative forcing, and ocean heat content anomalies relative to the preindustrial state. However, internal variability could lead to a systematic bias if the chosen period is in a phase of internal variability that leads to a particular bias in EffCS [an effect that is neglected in Otto et al. (2013)]. For example, Andrews et al. (2018) show that differences between the observed pattern of warming and the projected pattern of long-term warming lead to a bias toward lower EffCS in the historical period. This observed warming pattern anomaly is generally found to be consistent with model estimates of internal variability (Watanabe et al. 2021; Olonscheck et al. 2020), at least on decadal time scales. Furthermore, internal variability is the main reason why EffCS can be sensitive to the chosen averaging period, as discussed in Forster (2016) and Loeb et al. (2016). We therefore aim to understand how different modes of internal variability contribute to anomalies in EffCS.

\section{a. Impact of low-frequency internal variability on EffCS}

To show the impact of variability in LFCs 1-3 on EffCS, we compute the decadal-mean anomalies in GMST and GMTOA associated with a two-standard-deviation anomaly in each LFC (Figs. 11a-c). Based on Eq. (6), the corresponding anomalies in EffCS are diagonal lines in this space of GMST and GMTOA. LFC-1 and LFC-2, which both have large impacts on GMST at lag 0 (Figs. 11a,b), have correspondingly large impacts on EffCS of $0.21^{\circ}$ and $0.20^{\circ} \mathrm{C}$, respectively (in the multimodel mean). The more positive TOA anomalies associated with LFC-2 afford it a comparable impact on EffCS despite its smaller impact on GMST compared to LFC-1 (e.g., compare MPI-ESM1-2-HR between Figs. 11a and 11b). Anomalies are small enough that the impact of negative anomalies in these modes is approximately the negative of these values, such that the total spreads in EffCS due to LFC-1 and LFC-2 are each $\pm 0.2^{\circ} \mathrm{C}$ (cf. total spread of $\pm 0.65^{\circ} \mathrm{C}$ ). The models with the largest amplitude variability in these modes (EC-Earth3 for LFC-1 and BCC-CSM2-MR for LFC-2) can have impacts on EffCS up to 5 times as large.

The lag-0 impact of the PDO-like LFC-3 on GMST is small and varies in sign across models (Fig. 11c), therefore contemporaneous (lag 0) LFC-3 anomalies have a minimal impact on EffCS in most models. However, this does not capture the effect of GMST trends associated with LFC-3 on EffCS. We therefore analyze the change in GMST and GMTOA between the five years before and the five years after a one-standarddeviation anomaly in each LFC (right-hand side of Fig. 11). This captures decadal trends in GMST, GMTOA, and EffCS associated with each LFC. Qualitatively, this can also be thought of as the decadal global climate anomalies that would result from a positive LFC anomaly at the beginning of a decade and a negative anomaly at the end of a decade (i.e., the decadal anomalies associated with a two-standard-deviation trend in the LFC). All models show a reduction in the rate of global warming during positive PDV events (based on LFC3; Fig. 11f), as has been suggested to explain the so-called global warming hiatus (Meehl et al. 2011; Trenberth and Fasullo 2013; Kosaka and Xie 2013; England et al. 2014; Liu et al. 2016). Equivalently, a negative trend in LFC-3 is associated with a negative GMST anomaly. Many models show a small anomalous energy input into the Earth system in response to this cooling, such that LFC-3 does not have a big influence on EffCS in these models (Fig. 11f; e.g., BCC-ESM1). In a few models, including CESM2 and CESM2-WACCM, the radiative response is the opposite sign (a positive feedback). In this case, cooling trends associated with LFC-3 can induce moderate negative anomalies in EffCS.

LFC-1 and LFC-2 can similarly induce a negative EffCS anomaly in their decay phase in models where the GMTOA anomalies are highly asymmetric about lag 0 (Figs. 11d,e), such as NorESM2-MM. In other words, the positive phases of LFCs 1-3 are associated with trends toward larger negative TOA radiation anomalies and thus trends toward more-negative EffCS anomalies, particularly in the CMIP6 models that have the largest GMTOA change (toward more radiation out of the Earth system) between the five years before and the five years after a positive LFC anomaly. However, the decadal trends in GMST and EffCS associated with LFC-1 and LFC-2 are generally smaller than the contemporaneous anomalies in GMST and EffCS (cf. Figs. 11a,b).

\section{b. Impact of ENSO on EffCS}

To investigate the influence of ENSO on decadal variability in EffCS, we examine a similar diagram based on the Niño-3.4 index (Fig. 12). The lag-0 influence of Niño-3.4 on decadal-mean GMST and GMTOA is small, leading to a small influence on EffCS (Fig. 12a). This is partly the result of cancellation between anomalies associated with El Niño and La Niña, and the influence of Niño-3.4 on 2-yr-mean GMST and GMTOA is more than 5 times larger (than on decadal-mean GMST and GMTOA) in the multimodel mean (Fig. 12b; cf. Fig. 12a; note different $x$ - and $y$-axis scales).

Because the lead-lag relationship between Niño-3.4 and GMTOA is asymmetric about lag 0 (Fig. 5), the impact of Niño-3.4 on decadal trends in GMTOA (i.e., the change between the five years before and the five years after) is much larger than its impact on the contemporaneous decade (Fig. 12c; cf. Fig. 12a). The trend in GMTOA causes the EffCS to be much lower in the period following an El Niño or preceding a La Niña (when the Earth system is losing energy) than in the period preceding an El Niño or following a La Niña (when the Earth system is gaining energy). In this way, the timing of ENSO events within a decadal period can lead to EffCS anomalies of $\pm 0.14^{\circ} \mathrm{C}$ (in the multimodel mean). The end members of this 


\section{a}

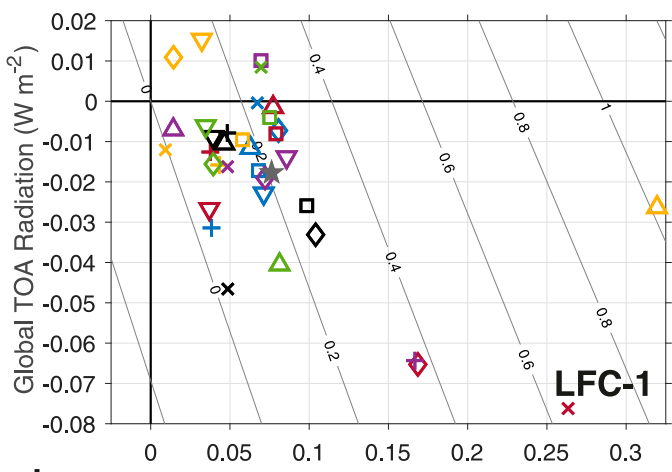

b

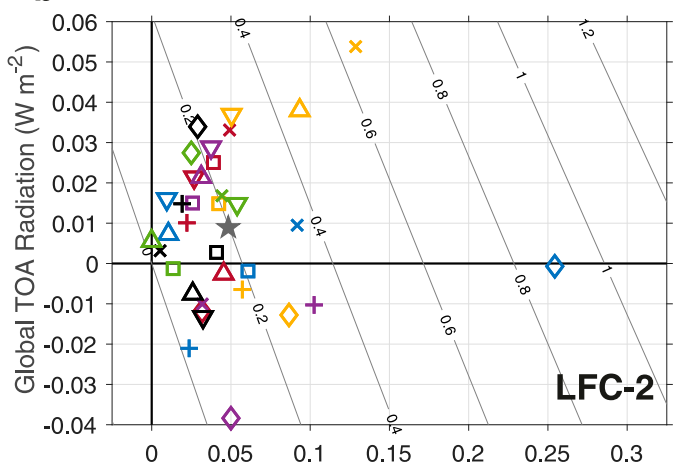

C

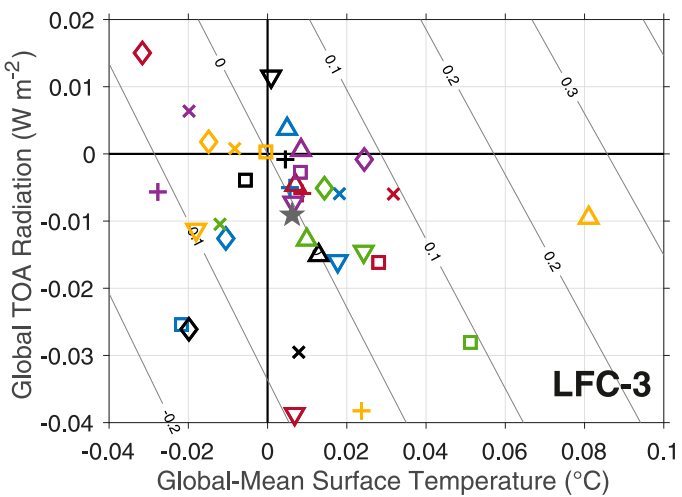

d Decadal Change (5 yr After - 5 yr Before)

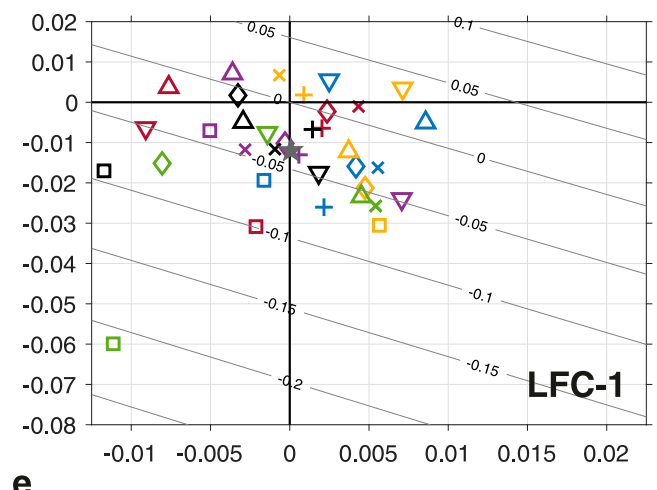

e
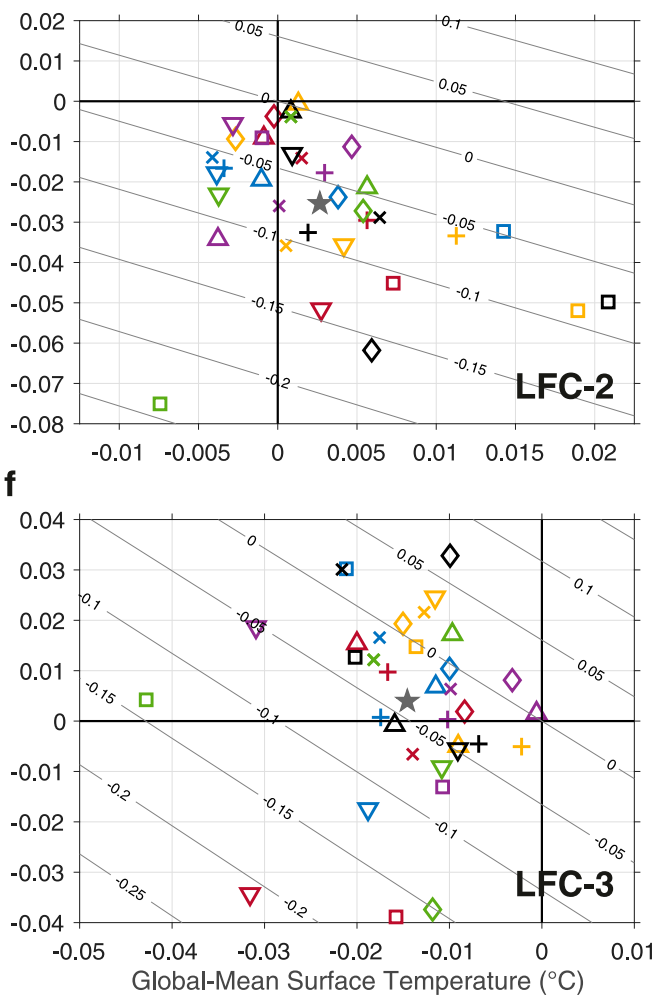

$\triangle$ ACCESS-CM2

$\nabla$ ACCESS-ESM1-5

AWI-CM-1-1-MR

$\diamond$ BCC-CSM2-MR

$\times$ BCC-ESM1

+ CAMS-CSM1-0

$\triangle$ CanESM5

$\nabla$ CESM2

口 CESM2-WACCM

$\diamond$ CNRM-CM6-1

$\times$ CNRM-ESM2-1

+ E3SM-1-0

$\triangle$ EC-Earth3

$\nabla$ FGOALS-f3-L

$\square$ FGOALS-g3

$\triangle$ GFDL-CM4

$\times$ GFDL-ESM4

+ GISS-E2-1-G

$\triangle$ GISS-E2-1-H

$\nabla$ HadGEM3-GC31-LL

r HadGEM3-GC31-MM

$\diamond$ INM-CM4-8

$\times$ INM-CM5-0

+ IPSL-CM6A-LR

$\triangle$ MIROC6

$\nabla$ MIROC-ES2L

I MPI-ESM-1-2-HAM

$\diamond$ MPI-ESM1-2-HR

$x$ MPI-ESM1-2-LR

+ MRI-ESM2-0

$\triangle$ NESM3

$\nabla$ NorCPM1

$\square$ NorESM2-MM

$\diamond$ SAMO-UNICON

$\times$ UKESM1-0-L

$\star$ Multi-Model Mean

FIG. 11. Scatterplots of the lag-0 (contemporaneous) impact of a two-standard-deviation anomaly in (a) LFC-1, (b) LFC-2, and (c) LFC-3 on 10-yr running-mean GMST ( $x$ axis) and 10-yr running-mean GMTOA ( $y$ axis) for all 35 of the CMIP6 piControl simulations. (d)-(f) The change in GMST ( $x$-axis) and GMTOA ( $y$ axis) between the 5 years before and 5 years after a one-standard-deviation anomaly in LFC-1, LFC-2, and LFC-3, respectively. Diagonal lines show the corresponding anomaly in effective climate sensitivity (EffCS) if these GMST and GMTOA anomalies are superimposed on a forced change of $1^{\circ} \mathrm{C}$ and $1.14 \mathrm{~W} \mathrm{~m}^{-2}$ (corresponding to an $\mathrm{EffCS}_{\text {forced }}$ of $3.5^{\circ} \mathrm{C}$ ).

95\% spread are decadal periods that begin at the peak of a onestandard-deviation El Niño event and end at the peak of a onestandard-deviation La Niña event, or vice versa. Note, however, that some of the impact of ENSO on decadal anomalies in GMST, GMTOA, and EffCS may come from the tendency for positive PDV anomalies to follow El Niño events (Newman et al. 2016; Wills et al. 2019b).

\section{c. Interpreting EffCS in historical periods}

Applying these insights on the sources of internal variability in EffCS to estimate the actual EffCS bias in a particular historical period would require further methodological developments (e.g., using detection and attribution methods to track changes in modes of variability over time) that are beyond the scope of this paper. Furthermore, the interpretation of the recent historical record through the lens of CMIP model variability must be made with caution, as the variability sampled by the CMIP ensemble may not adequately capture historical variations in SSTs (Cane et al. 1997; Laepple and Huybers 2014; Coats and Karnauskas 2017), the Walker circulation and trade winds (L'Heureux et al. 2013; Kociuba and Power 2015), or radiative feedbacks (Zhou et al. 2016; Andrews et al. 2018). 
a

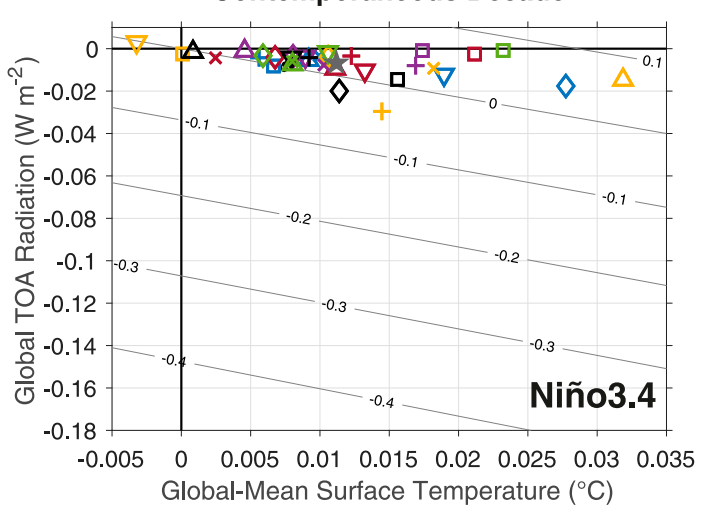

b Contemporaneous 2 Years

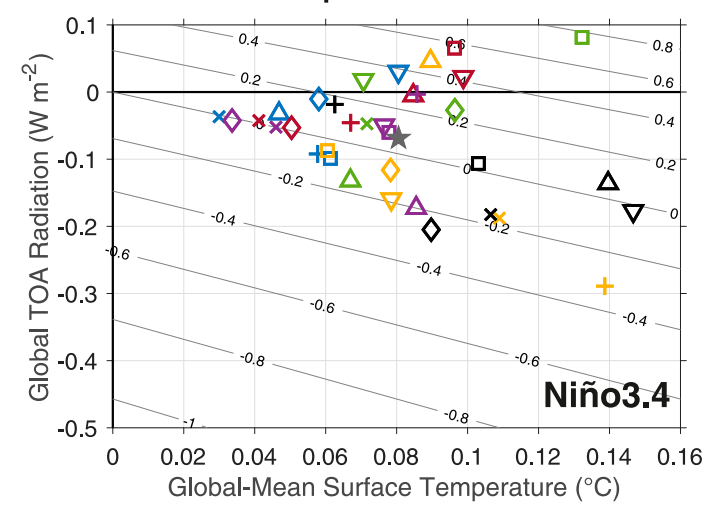

C Decadal Change (Decay Phase - Growth Phase)

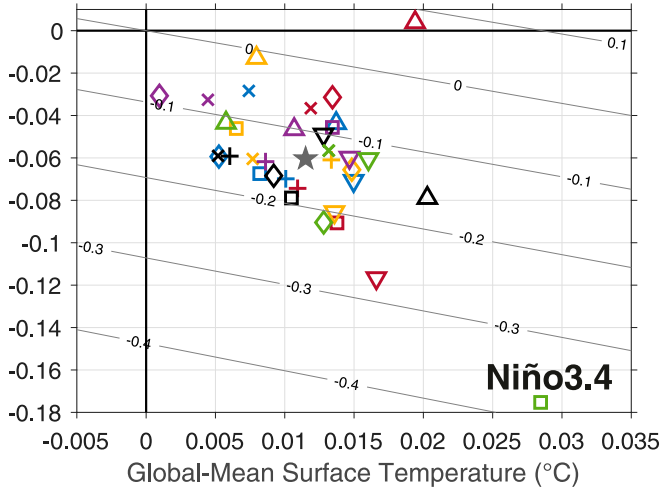

d 2-yr Change (Decay Phase - Growth Phase)

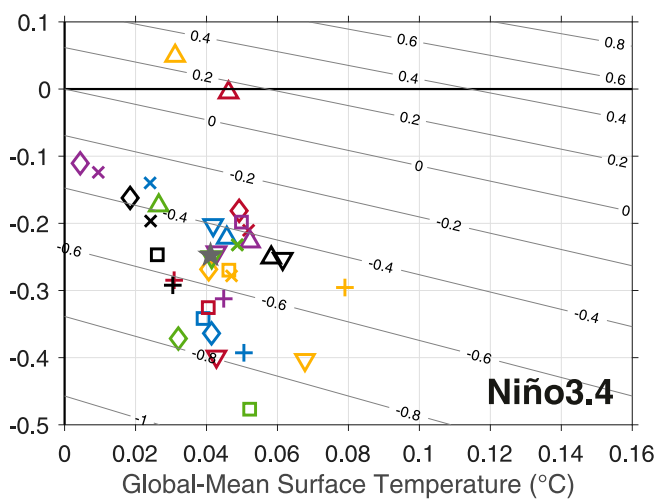

$\triangle$ ACCESS-CM2

$\nabla$ ACCESS-ESM1-5

口 AWI-CM-1-1-MR

$\diamond$ BCC-CSM2-MR

$\times$ BCC-ESM1

+ CAMS-CSM1-0

$\triangle$ CanESM5

$\nabla$ CESM2

C CESM2-WACCM

$\diamond$ CNRM-CM6-1

$\times$ CNRM-ESM2-1

+ E3SM-1-0

$\triangle$ EC-Earth3

$\nabla$ FGOALS-f3-L

I FGOALS-g3

GFDL-CM4

$\times$ GFDL-ESM4

+ GISS-E2-1-G

$\triangle$ GISS-E2-1-H

$\nabla$ HadGEM3-GC31-LL

口 HadGEM3-GC31-MM

$\diamond$ INM-CM4-8

$\times \quad$ INM-CM5-0

+ IPSL-CM6A-LR

$\triangle$ MIROC6

$\nabla$ MIROC-ES2L

MPI-ESM-1-2-HAM

$\diamond$ MPI-ESM1-2-HR

$\times$ MPI-ESM1-2-LR

+ MRI-ESM2-0

$\triangle$ NESM3

$\nabla$ NorCPM1

- NorESM2-MM

$\checkmark$ SAMO-UNICON

$\times$ UKESM1-0-L

$\star$ Multi-Model Mean

FIG. 12. (a) Scatterplots of the lag-0 (contemporaneous) impact of a two-standard-deviation anomaly in Niño-3.4 on 10-yr running-mean GMST ( $x$ axis) and 10-yr running-mean GMTOA ( $y$ axis) for all 35 of the CMIP6 piControl simulations. (b) As in (a), but for 2-yr running means. (c) The change in GMST ( $x$ axis) and GMTOA ( $y$ axis) between the 5 years before and 5 years after a two-standard-deviation anomaly in Niño-3.4. (d) As in (c), but for 1-yr averages. Diagonal lines show the corresponding anomaly in effective climate sensitivity (EffCS) if these GMST and GMTOA anomalies are superimposed on a forced change of $1^{\circ} \mathrm{C}$ and $1.14 \mathrm{~W} \mathrm{~m}^{-2}$ (corresponding to an $\mathrm{EffCS}_{\text {forced }}$ of $3.5^{\circ} \mathrm{C}$ ).

Nevertheless, we can provide some qualitative arguments for how internal variability may have influenced recent climate sensitivity estimates from observations.

During the time periods used by Otto et al. (2013) (2000-09) and Lewis and Curry (2015) (1995-2011), the high latitudes of the North Atlantic and Arctic had warmed more than projected in uninitialized climate model simulations (Yeager et al. 2015), while the Southern Ocean had warmed less than projected (Armour et al. 2016). These anomalies correspond to a positive anomaly in LFC-1 and a negative anomaly in LFC-2, which would lead to large positive and negative EffCS anomalies, respectively. However, the extent to which these anomalies resulted purely from internal variability (versus differences in the forced response between observations and models) is uncertain. If these anomalies are due to internal variability, the EffCS anomalies would be interpreted as differences between EffCS and EffCS $_{\text {forced; }}$ if they are due to an incorrect forced response in models, the EffCS anomalies would be interpreted as differences in $\mathrm{EffCS}_{\text {forced }}$ between the real world and models. This highlights the need for better methods to separate forced and unforced components of climate change in observations.

There were also large changes in Niño-3.4 during these two time periods; the $2000-09$ period started from a moderate La
Niña and ended with a moderate El Niño, whereas the 19952011 period started from a weak El Niño and ended with a weak La Niña. Our analysis of the global energy budget impacts of Niño-3.4 suggests that these ENSO anomalies at the beginning and end of these time periods could lead to opposite impacts on EffCS, with a positive EffCS bias based on 2000-09 and a negative bias based on 1995-2011. The general agreement between these two estimates, however, suggests that this was not the case. The full time evolution of Niño-3.4 during these time periods (rather than just the anomalies at the end points) could be important in determining the anomalies in the global energy budget and the corresponding anomalies in EffCS. Together, the large global radiative impacts of ENSO and the difficulties in capturing these impacts with a simple linear regression approach suggest that great caution is needed in estimating climate sensitivity from short observational records.

\section{Summary and conclusions}

In this paper, we showed that CMIP6 models have largeamplitude interdecadal GMST variability resulting from variability in the high-latitude oceans. Interdecadal variability in 
the Northern Hemisphere (i.e., LFC-1) is closely tied to the AMV, with large SST anomalies in the North Atlantic subpolar gyre associated with coupled atmosphere-ocean variability (Wills et al. 2019a and references therein). Interdecadal variability in the Southern Hemisphere is dominated by zonally symmetric SST changes in the Southern Ocean (i.e., LFC-2). In models where LFC-2 variability is strongest, it is linked to variability in open-ocean deep convection (Martin et al. 2013; Pedro et al. 2016; Behrens et al. 2016; Reintges et al. 2017; Zhang et al. 2017; Cabré et al. 2017). The large area of SST anomalies associated with LFC-1 and LFC-2 as well as their impacts on sea ice extent and Arctic/Antarctic surface air temperatures afford them a large impact on GMST. The long time scale of these modes of variability means that the associated GMST anomalies can persist for multiple decades (Fig. 1) or even up to a century in some models (Parsons et al. 2020).

Despite their large impact on GMST, neither of these modes has a large impact on the global energy balance at TOA. In both cases, large, negative longwave radiative feedbacks, which would act to damp GMST anomalies, are cancelled by positive shortwave radiative feedbacks, such as the sea icealbedo feedback and low-cloud feedback. These cancelling feedbacks result in these modes having weak global radiative feedbacks of approximately $-0.5 \mathrm{~W} \mathrm{~m}^{-2} \mathrm{~K}^{-1}$. If such low global radiative feedbacks occurred in response to a doubling of $\mathrm{CO}_{2}$ (something that should only be considered a thought experiment), then this would correspond to an ECS of $\sim 8^{\circ} \mathrm{C}$.

Cancellation between longwave and shortwave feedbacks also occurs in PDV-like LFC-3 variability, except in the year before and the year after its peak. As a consequence, LFC-3 also has a weak global radiative feedback, though not as weak as LFC-1 and LFC-2. In contrast, longwave and shortwave feedbacks associated with ENSO reinforce each other at most lead and lag times, leading to large impacts on the TOA energy balance and a correspondingly large global radiative feedback of approximately $-3 \mathrm{~W} \mathrm{~m}^{-2} \mathrm{~K}^{-1}$. ENSO is therefore the mode of variability with the largest impact on GMTOA out to decadal time scales, despite its predominantly interannual time scale. The strong global radiative feedbacks associated with ENSO variability act to damp the associated GMST anomalies and prevent them from persisting for more than a year or two.

A further takeaway of our analysis is that there is a diverse array of possible lead-lag relationships between modes of variability and global climate impacts. While GMST anomalies develop in proportion to LFC-1 and LFC-2 anomalies and (with a few months lag) to ENSO anomalies, this is not the case for the PDV-like LFC-3, which is associated instead with an anomaly in the rate of GMST change. GMTOA anomalies exhibit an even wider range of lead-lag relationships with the modes of variability. In the case of LFC-1 and LFC-2, weakly negative GMTOA anomalies develop after their warm phases. However, LFC-2 also shows positive GMTOA anomalies in the decades leading up to its warm phase, evidence that positive radiative feedbacks (e.g., the sea ice-albedo feedback) act to amplify its growth. LFC-3 and ENSO radiative anomalies have a similar temporal evolution to their respective GMST anomalies, but with opposite signs and slight lags. In both cases, GMTOA anomalies near the peak of the LFC-3 or ENSO event act to change the sign of GMST anomalies. In the case of ENSO, the relationship between GMTOA and GMST can be modeled with a stochastically forced linear oscillator (Proistosescu et al. 2018).

Internal variability in GMST and GMTOA can lead to biases in estimates of the global climate feedback from short time periods, as have been used to estimate climate sensitivity from observations (Otto et al. 2013; Lewis and Curry 2015; Forster 2016; Knutti et al. 2017; Sherwood et al. 2020). We found that decadal internal variability of the magnitude found in CMIP6 piControl simulations can lead to biases of $\pm 0.65^{\circ} \mathrm{C}$ $\left( \pm 0.36^{\circ} \mathrm{C}\right)$ in the effective climate sensitivity (EffCS) inferred from 10-yr (30-yr) average anomalies, when superimposed on externally forced GMST and global energy budget changes of $\bar{T}_{\text {forced }}=1^{\circ} \mathrm{C}$ and $\bar{F}-\bar{N}_{\text {forced }}=1.14 \mathrm{~W} \mathrm{~m}^{-2}$. The spread in EffCS due to internal variability would be larger for smaller forced temperature changes or for smaller global radiative feedbacks (larger effective climate sensitivities). The biggest contributors to the spread in EffCS are the slow modes of global temperature variability in the high latitudes (LFC-1 and LFC-2), which can each lead to multimodel median biases in EffCS (based on 10-yr average anomalies) of approximately $\pm 0.2^{\circ} \mathrm{C}$ and biases up to 5 times as large in some models (Figs. 11a,b). The PDV-like LFC-3 can lead to anomalies in the rate of GMST change, but does not lead to large anomalies in EffCS in most models.

The complex phase relationships between modes of variability, GMST, and GMTOA mean that a detailed analysis of the contribution of modes of internal variability to EffCS biases in an individual decade requires a careful treatment of the phase evolution of modes of variability. In particular, we find that it is important to capture the full evolution of ENSO events within an averaging period, because EffCS will otherwise be strongly biased due to large differences in the global energy budget between the El Niño growth and decay phases. These timeevolving global energy budget anomalies can lead to EffCS biases of approximately $\pm 0.14^{\circ} \mathrm{C}$ if the decadal averaging period used starts from a one-standard-deviation La Niña event and ends with a one-standard-deviation El Niño event, or vice versa.

Some important caveats of our study are that our analysis is entirely based upon CMIP6 models, which struggle to reproduce observed multidecadal SST changes (Laepple and Huybers 2014; Watanabe et al. 2021), that we only considered a subset of the diverse modes of climate variability, that we did not consider the potential nonlinear superposition of radiative impacts from different modes of variability, and that we made no distinction between anomalies in different seasons. Further work is needed to validate some of our conclusions in observations. In particular, longer records and/or better methods to remove the forced climate response are needed to determine the magnitude of decadal internal variability in observations, especially in the North Atlantic and Southern Ocean. The radiative impacts of these slow modes of high-latitude variability are hard to verify in observations until we have longer records of TOA radiation. A more thorough treatment of the seasonality of global energy budget anomalies may also give further insight into the mechanisms of variability in global climate.

Overall, we have shown that modes of interdecadal SST variability in CMIP6 models are concentrated in the high latitudes, where they have a large impact on GMST but a minimal impact 
on GMTOA. The largest and most persistent GMST anomalies result from variability in North Atlantic SSTs linked to AMOC or variability in Southern Ocean SSTs linked to poleward ocean heat transport. The minimal impact of these slow modes of global temperature variability on GMTOA results from the cancelation between positive shortwave cloud and sea ice-albedo feedbacks and the negative feedback of outgoing longwave radiation. This suggests that externally forced warming in the high latitudes is also likely to be weakly damped by radiation, such that a greater degree of polar amplification could be associated with a higher climate sensitivity. Tropical SST variability, such as that associated with ENSO, is generally associated with large impacts on GMTOA. However, subtle differences in the SST pattern between the build up and decay phases of El Niño can give rise to opposite-signed anomalies in GMTOA, suggesting that the climate sensitivity in response to external forcing may depend sensitively on the details of the tropical SST pattern [consistent with the work of Dong et al. (2019)].

We hypothesize that it is not a coincidence that the slowest modes of global temperature variability are in regions of weak radiative feedbacks. Rather, we suggest that the lack of strong radiative damping in the polar regions, where anomalies remain trapped beneath a strong inversion, as well as the relative inefficiency of atmospheric heat transport away from these regions (Stuecker et al. 2018; Armour et al. 2019), may be the reason that SST anomalies in these regions can persist for several decades. In contrast, SST anomalies in regions of tropical deep convection will quickly be communicated to the free troposphere, where they can influence the global lapse rate and cloud fraction and lead to strong radiative damping (Zhou et al. 2017; Ceppi and Gregory 2017; Dong et al. 2019). As a consequence, SST anomalies in tropical deep convective regions result in a large loss of energy at the global scale. In the absence of a large source of energy (e.g., strong energy exchange with the deep ocean), these tropical SST anomalies would not be able to persist on decadal time scales. We therefore suggest that tropical SST anomalies will generally be associated with large changes in Earth's energy budget, whether forced or unforced.

Acknowledgments. R.C.J.W. and D.S.B. acknowledge support from the National Science Foundation (Grant AGS-1929775) and the Tamaki Foundation. R.C.W. and K.C.A. acknowledge support from the National Science Foundation (Grant AGS1752796). C.P. acknowledges support from the National Science Foundation (Grant OCE-2002385). K.C.A. and C.P. acknowledge support from the National Oceanic and Atmospheric Administration MAPP Program (Award NA20OAR4310391). L. Parsons acknowledges support from NASA GISS Grant 80NSSC19M0138. We thank Maria Rugenstein, Jonah Bloch-Johnson, Aaron Donohoe, and three anonymous reviewers for valuable input on this work. We acknowledge the World Climate Research Programme, which, through its Working Group on Coupled Modelling, coordinated and promoted CMIP6. We thank the climate modeling groups for producing and making available their model output, the Earth System Grid Federation (ESGF) for archiving the data and providing access, and the multiple funding agencies who support CMIP6 and ESGF. We thank Peter Huybers for providing scripts

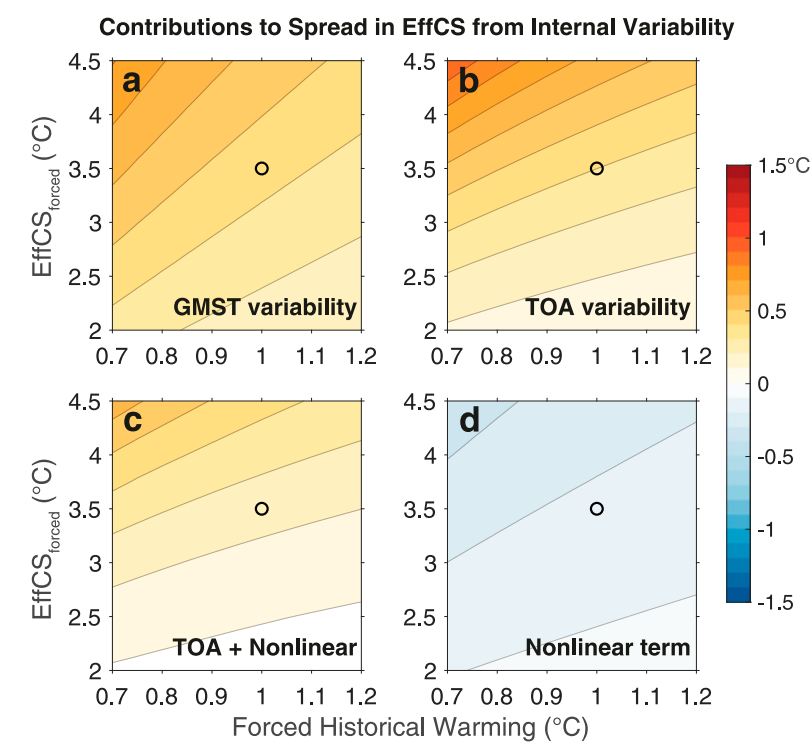

FIG. A1. Contributions to the two-standard-deviation $(\approx 95 \%)$ spread in effective climate sensitivity (EffCS), calculated from 10yr running averages, from internal variability in (a) GMST, (b) GMTOA, (c) GMTOA and nonlinear terms that depend on the covariance of GMST and GMTOA, and (d) the nonlinear terms that depend on the covariance of GMST and GMTOA; i.e., (c) = (b) + (d). This calculation is based on the superposition of internal variability from CMIP6 piControl simulations, an assumed externally forced historical warming $\left(\bar{T}_{\text {forced }} ; x\right.$ axis), and an assumed radiative response corresponding a forced effective climate sensitivity ( $\mathrm{EffCS}_{\text {forced }} ; y$ axis). Values are the median across 35 CMIP6 models.

for the estimation of spectra and coherence using a multi-taper method, which are available at https://www.people.fas.harvard.edu/ phuybers/Mfiles/. The analysis code and processed CMIP6 data associated with this paper can be found at https:/github.com/ rcjwills/cmip6-global-lfca/.

\section{APPENDIX}

\section{Contributions to EffCS Spread}

Here, we provide expressions for the contributions of internal variability in GMST ( $\left.\bar{T}_{\text {internal }}\right)$ and GMTOA $\left(\bar{N}_{\text {internal }}\right)$ to anomalies in EffCS. We start from the full expression for EffCS:

$$
\text { EffCS }=-\frac{\bar{F}_{2 \mathrm{xCO}_{2}}\left(\bar{T}_{\text {forced }}+\bar{T}_{\text {internal }}\right)}{\left(\bar{R}_{\text {forced }}+\bar{N}_{\text {internal }}\right)} .
$$

For conciseness, we have introduced $\bar{R}_{\text {forced }}=\bar{N}_{\text {forced }}-\bar{F}_{\text {obs }}$. The effective climate sensitivity associated with the forced response is determined by setting $\bar{T}_{\text {internal }}$ and $\bar{N}_{\text {internal }}$ to zero:

$$
\mathrm{EffCS}_{\text {forced }} \equiv-\frac{\bar{F}_{2 \mathrm{xCO}} \bar{T}_{\text {forced }}}{\bar{R}_{\text {forced }}} .
$$

The difference between EffCS and EffCS $_{\text {forced }}$ is split into three components, due to $\bar{T}_{\text {internal }}, \bar{N}_{\text {internal }}$, or a combination of the two: 


$$
\begin{aligned}
& \operatorname{EffCS}_{\mathrm{GMST}} \equiv-\frac{\bar{F}_{2 \mathrm{xCO}_{2}}\left(\bar{T}_{\text {forced }}+\bar{T}_{\text {internal }}\right)}{\bar{R}_{\text {forced }}}-\mathrm{EffCS}_{\text {forced }} \\
& =-\frac{\bar{F}_{2 \mathrm{xCO}_{2}} \bar{T}_{\text {internal }}}{\bar{R}_{\text {forced }}}, \\
& \text { EffCS }_{\mathrm{TOA}} \equiv-\frac{\bar{F}_{2 \times \mathrm{CO}_{2}} \bar{T}_{\text {forced }}}{\left(\bar{R}_{\text {forced }}+\bar{N}_{\text {internal }}\right)}-\text { EffCS }_{\text {forced }} \\
& =\frac{\bar{F}_{2 \mathrm{xCO}_{2}} \bar{T}_{\text {forced }} \bar{N}_{\text {internal }}}{\bar{R}_{\text {forced }}\left(\bar{R}_{\text {forced }}+\bar{N}_{\text {internal }}\right)}, \\
& \text { EffCS }_{\text {nonlinear }} \equiv \text { EffCS }- \text { EffCS }_{\text {forced }} \\
& - \text { EffCS }_{\mathrm{GMST}}-\text { EffCS }_{\mathrm{TOA}} \text {. } \\
& =\frac{\bar{F}_{2 \mathrm{xCO}} \bar{T}_{\text {internal }} \bar{N}_{\text {internal }}}{\bar{R}_{\text {forced }}\left(\bar{R}_{\text {forced }}+\bar{N}_{\text {internal }}\right)} \text {. }
\end{aligned}
$$

For conciseness, we have omitted several steps of straightforward algebra. The total EffCS spread shown in Fig. 10a is split into these three terms in Figs. A1a, A1b, and A1d, respectively. Figure A1c shows the combination of EffCS $_{\mathrm{TOA}}$ and $\mathrm{EffCS}_{\text {nonlinear }}$ (i.e., all terms that depend on $\left.\bar{N}_{\text {internal }}\right)$. The contribution to the spread in EffCS from $\bar{T}_{\text {internal }}$ (Fig. A1a) is larger than the contribution from $\bar{N}_{\text {internal }}$ (including the nonlinear term; Fig A1c) for all values of the forced response; it is much larger in cases where historical global warming is large or EffCS $_{\text {forced }}$ is small.

\section{REFERENCES}

Adames, Á. F., and J. M. Wallace, 2017: On the tropical atmospheric signature of El Niño. J. Atmos. Sci., 74, 1923-1939, https://doi.org/10.1175/JAS-D-16-0309.1.

Alexander, M. A., C. Deser, and M. S. Timlin, 1999: The reemergence of SST anomalies in the North Pacific Ocean. J. Climate, 12, 2419-2433, https:/doi.org/10.1175/1520-0442(1999)012<2419:TROSAI>2.0.CO;2. , I. Bladé, M. Newman, J. R. Lanzante, N.-C. Lau, and J. D. Scott, 2002: The atmospheric bridge: The influence of ENSO teleconnections on air-sea interaction over the global oceans. J. Climate, 15, 2205-2231, https://doi.org/10.1175/1520-0442(2002) 015<2205:TABTIO>2.0.CO;2.

Andrews, T., J. M. Gregory, and M. J. Webb, 2015: The dependence of radiative forcing and feedback on evolving patterns of surface temperature change in climate models. J. Climate, 28, 1630-1648, https://doi.org/10.1175/JCLI-D-14-00545.1.

— , and Coauthors, 2018: Accounting for changing temperature patterns increases historical estimates of climate sensitivity. Geophys. Res. Lett., 45, 8490-8499, https://doi.org/10.1029/2018GL078887.

Armour, K. C., C. M. Bitz, and G. H. Roe, 2013: Time-varying climate sensitivity from regional feedbacks. J. Climate, 26, 4518-4534, https://doi.org/10.1175/JCLI-D-12-00544.1.

— J. Marshall, J. R. Scott, A. Donohoe, and E. R. Newsom, 2016: Southern Ocean warming delayed by circumpolar upwelling and equatorward transport. Nat. Geosci., 9, 549-554, https://doi.org/10.1038/ngeo2731.

— , N. Siler, A. Donohoe, and G. H. Roe, 2019: Meridional atmospheric heat transport constrained by energetics and mediated by large-scale diffusion. J. Climate, 32, 3655-3680, https://doi.org/10.1175/JCLI-D-18-0563.1.

Behrens, E., G. Rickard, O. Morgenstern, T. Martin, A. Osprey, and M. Joshi, 2016: Southern Ocean deep convection in global climate models: A driver for variability of subpolar gyres and Drake Passage transport on decadal timescales. J. Geophys. Res. Oceans, 121, 3905-3925, https://doi.org/10.1002/2015JC011286.

Bellomo, K., A. Clement, T. Mauritsen, G. Rädel, and B. Stevens, 2014: Simulating the role of subtropical stratocumulus clouds in driving Pacific climate variability. J. Climate, 27, 5119-5131, https://doi.org/10.1175/JCLI-D-13-00548.1.

,- L. N. Murphy, L. M. Polvani, and M. A. Cane, 2016: New observational evidence for a positive cloud feedback that amplifies the Atlantic multidecadal oscillation. Geophys. Res. Lett., 43, 9852-9859, https://doi.org/10.1002/2016GL069961.

Bloch-Johnson, J., M. Rugenstein, and D. S. Abbot, 2020: Spatial radiative feedbacks from internal variability using multiple regression. J. Climate, 33, 4121-4140, https://doi.org/10.1175/ JCLI-D-19-0396.1.

Bond, N., J. Overland, M. Spillane, and P. Stabeno, 2003: Recent shifts in the state of the North Pacific. Geophys. Res. Lett., 30, 2183, https://doi.org/10.1029/2003GL018597.

Broccoli, A. J., K. A. Dahl, and R. J. Stouffer, 2006: Response of the ITCZ to Northern Hemisphere cooling. Geophys. Res. Lett., 33, L01702, https://doi.org/10.1029/2005GL024546.

Brown, P. T., W. Li, L. Li, and Y. Ming, 2014: Top-of-atmosphere radiative contribution to unforced decadal global temperature variability in climate models. Geophys. Res. Lett., 41, 51755183, https://doi.org/10.1002/2014GL060625.

,-- , and S.-P. Xie, 2015: Regions of significant influence on unforced global mean surface air temperature variability in climate models. J. Geophys. Res., 120, 480-494, https://doi.org/ 10.1002/2014JD022576.

—, M. S. Lozier, R. Zhang, and W. Li, 2016: The necessity of cloud feedback for a basin-scale Atlantic Multidecadal Oscillation. Geophys. Res. Lett., 43, 3955-3963, https://doi.org/10.1002/ 2016 GL068303.

Cabré, A., I. Marinov, and A. Gnanadesikan, 2017: Global atmospheric teleconnections and multidecadal climate oscillations driven by Southern Ocean convection. J. Climate, 30, 81078126, https://doi.org/10.1175/JCLI-D-16-0741.1.

Cane, M. A., A. C. Clement, A. Kaplan, Y. Kushnir, D. Pozdnyakov, R. Seager, S. E. Zebiak, and R. Murtugudde, 1997: Twentiethcentury sea surface temperature trends. Science, 275, 957-960, https://doi.org/10.1126/science.275.5302.957.

Capotondi, A., and Coauthors, 2015: Understanding ENSO diversity. Bull. Amer. Meteor. Soc., 96, 921-938, https://doi.org/ 10.1175/BAMS-D-13-00117.1.

Ceppi, P., and J. M. Gregory, 2017: Relationship of tropospheric stability to climate sensitivity and Earth's observed radiation budget. Proc. Natl. Acad. Sci. USA, 114, 13 126-13131, https:// doi.org/10.1073/pnas.1714308114.

Charney, J. G., and Coauthors, 1979: Carbon Dioxide and Climate: A Scientific Assessment. National Academy of Sciences, 22 pp.

Chen, X., and K.-K. Tung, 2014: Varying planetary heat sink led to global-warming slowdown and acceleration. Science, 345, 897903, https://doi.org/10.1126/science.1254937.

_- , and J. M. Wallace, 2016: Orthogonal PDO and ENSO indices. J. Climate, 29, 3883-3892, https://doi.org/10.1175/ JCLI-D-15-0684.1.

—_ and K.-K. Tung, 2017: Global-mean surface temperature variability: Space-time perspective from rotated EOFs. Climate Dyn., 51, 1719-1732, https://doi.org/10.1007/s00382-017-3979-0.

Chiang, J. C., and D. J. Vimont, 2004: Analogous Pacific and Atlantic meridional modes of tropical atmosphere-ocean variability. J. Climate, 17, 4143-4158, https://doi.org/10.1175/ JCLI4953.1. 
- and C. M. Bitz, 2005: Influence of high latitude ice cover on the marine Intertropical Convergence Zone. Climate Dyn., 25, 477-496, https://doi.org/10.1007/s00382-005-0040-5.

— S. E. Zebiak, and M. A. Cane, 2001: Relative roles of elevated heating and surface temperature gradients in driving anomalous surface winds over tropical oceans. J. Atmos. Sci., 58, 1371-1394, https://doi.org/10.1175/1520-0469(2001)058<1371: RROEHA $>2.0 . \mathrm{CO} ; 2$.

Coats, S., and K. Karnauskas, 2017: Are simulated and observed twentieth century tropical Pacific sea surface temperature trends significant relative to internal variability? Geophys. Res. Lett., 44, 9928-9937, https://doi.org/10.1002/2017GL074622.

DelSole, T., 2001: Optimally persistent patterns in time-varying fields. J. Atmos. Sci., 58, 1341-1356, https://doi.org/10.1175/ 1520-0469(2001)058<1341:OPPITV $>2.0 . C O ; 2$.

_- M. K. Tippett, and J. Shukla, 2011: A significant component of unforced multidecadal variability in the recent acceleration of global warming. J. Climate, 24, 909-926, https://doi.org/ 10.1175/2010JCLI3659.1.

Déqué, M., 1988: 10-day predictability of the Northern Hemisphere winter $500-\mathrm{mb}$ height by the ECMWF operational model. Tellus, 40A, 26-36, https://doi.org/10.3402/tellusa.v40i1.11780.

Deser, C., 2000: On the teleconnectivity of the "Arctic Oscillation." Geophys. Res. Lett., 27, 779-782, https://doi.org/ 10.1029/1999GL010945.

__, and J. M. Wallace, 1990: Large-scale atmospheric circulation features of warm and cold episodes in the tropical Pacific. J. Climate, 3, 1254-1281, https://doi.org/10.1175/1520-0442(1990) $003<1254:$ LSACFO $>2$.0.CO;2.

Dessler, A. E., 2013: Observations of climate feedbacks over 200010 and comparisons to climate models. J. Climate, 26, 333-342, https://doi.org/10.1175/JCLI-D-11-00640.1.

_- T. Mauritsen, and B. Stevens, 2018: The influence of internal variability on Earth's energy balance framework and implications for estimating climate sensitivity. Atmos. Chem. Phys., 18, 5147-5155, https://doi.org/10.5194/acp-18-5147-2018.

Di Lorenzo, E., and Coauthors, 2008: North Pacific Gyre Oscillation links ocean climate and ecosystem change. Geophys. Res. Lett., 35, L08607, https://doi.org/10.1029/2007GL032838.

Dong, Y., C. Proistosescu, K. C. Armour, and D. S. Battisti, 2019: Attributing historical and future evolution of radiative feedbacks to regional warming patterns using a Green's function approach: The preeminence of the western Pacific. J. Climate, 32, 5471-5491, https://doi.org/10.1175/JCLI-D-18-0843.1.

England, M. H., and Coauthors, 2014: Recent intensification of wind-driven circulation in the Pacific and the ongoing warming hiatus. Nat. Climate Change, 4, 222-227, https://doi.org/ 10.1038/nclimate2106.

Eyring, V., S. Bony, G. A. Meehl, C. A. Senior, B. Stevens, R. J. Stouffer, and K. E. Taylor, 2016: Overview of the Coupled Model Intercomparison Project Phase 6 (CMIP6) experimental design and organization. Geosci. Model Dev., 9, 19371958, https://doi.org/10.5194/gmd-9-1937-2016.

Forster, P. M., 2016: Inference of climate sensitivity from analysis of Earth's energy budget. Annu. Rev. Earth Planet. Sci., 44, 85106, https://doi.org/10.1146/annurev-earth-060614-105156.

Frankcombe, L. M., M. H. England, M. E. Mann, and B. A. Steinman, 2015: Separating internal variability from the externally forced climate response. J. Climate, 28, 8184-8202, https://doi.org/10.1175/JCLI-D-15-0069.1.

Gregory, J., and Coauthors, 2004: A new method for diagnosing radiative forcing and climate sensitivity. Geophys. Res. Lett., 31, L03205, https://doi.org/10.1029/2003GL018747.
Johnson, G. C., and A. N. Birnbaum, 2017: As El Niño builds, Pacific warm pool expands, ocean gains more heat. Geophys. Res. Lett., 44, 438-445, https://doi.org/10.1002/2016GL071767.

Kaiser, H. F., 1958: The varimax criterion for analytic rotation in factor analysis. Psychometrika, 23, 187-200, https://doi.org/ 10.1007/BF02289233.

Keenlyside, N., M. Latif, J. Jungclaus, L. Kornblueh, and E. Roeckner, 2008: Advancing decadal-scale climate prediction in the North Atlantic sector. Nature, 453, 84-88, https://doi.org/10.1038/nature06921.

Knutti, R., M. A. Rugenstein, and G. C. Hegerl, 2017: Beyond equilibrium climate sensitivity. Nat. Geosci., 10, 727-736, https://doi.org/10.1038/ngeo3017.

Kociuba, G., and S. B. Power, 2015: Inability of CMIP5 models to simulate recent strengthening of the Walker circulation: Implications for projections. J. Climate, 28, 20-35, https:// doi.org/10.1175/JCLI-D-13-00752.1.

Kosaka, Y., and S.-P. Xie, 2013: Recent global-warming hiatus tied to equatorial Pacific surface cooling. Nature, 501, 403-407, https://doi.org/10.1038/nature12534.

Laepple, T., and P. Huybers, 2014: Global and regional variability in marine surface temperatures. Geophys. Res. Lett., 41, 25282534, https://doi.org/10.1002/2014GL059345.

Latif, M., T. Martin, and W. Park, 2013: Southern Ocean sector centennial climate variability and recent decadal trends. J. Climate, 26, 7767-7782, https://doi.org/10.1175/JCLI-D-12-00281.1.

Lewis, N., and J. A. Curry, 2015: The implications for climate sensitivity of AR5 forcing and heat uptake estimates. Climate Dyn., 45, 1009-1023, https://doi.org/10.1007/s00382-014-2342-y.

L'Heureux, M. L., S. Lee, and B. Lyon, 2013: Recent multidecadal strengthening of the Walker circulation across the tropical Pacific. Nat. Climate Change, 3, 571-576, https://doi.org/ 10.1038/nclimate1840.

Li, Z., W. Zhang, F.-F. Jin, M. F. Stuecker, C. Sun, A. F. Levine, H. Xu, and C. Liu, 2020: A robust relationship between multidecadal global warming rate variations and the Atlantic multidecadal variability. Climate Dyn., 55, 1945-1959, https:// doi.org/10.1007/s00382-020-05362-8.

Liu, W., S.-P. Xie, and J. Lu, 2016: Tracking ocean heat uptake during the surface warming hiatus. Nat. Commun., 7, 10926, https://doi.org/10.1038/ncomms10926.

Loeb, N. G., W. Su, and S. Kato, 2016: Understanding climate feedbacks and sensitivity using observations of Earth's energy budget. Curr. Climate Change Rep., 2, 170-178, https://doi.org/ 10.1007/s40641-016-0047-5.

Lutsko, N. J., 2018: The relationship between cloud radiative effect and surface temperature variability at El Niño-Southern Oscillation frequencies in CMIP5 models. Geophys. Res. Lett., 45, 10-599, https://doi.org/10.1029/2018GL079236.

_ , and K. Takahashi, 2018: What can the internal variability of CMIP5 models tell us about their climate sensitivity? J. Climate, 31, 5051-5069, https://doi.org/10.1175/JCLI-D-17-0736.1.

Mantua, N. J., S. R. Hare, Y. Zhang, J. M. Wallace, and R. C. Francis, 1997: A Pacific interdecadal climate oscillation with impacts on salmon production. Bull. Amer. Meteor. Soc., 78, 1069-1080, https://doi.org/10.1175/1520-0477(1997)078<1069: APICOW $>2.0 . \mathrm{CO} ; 2$.

Martin, T., W. Park, and M. Latif, 2013: Multi-centennial variability controlled by Southern Ocean convection in the Kiel Climate Model. Climate Dyn., 40, 2005-2022, https://doi.org/ 10.1007/s00382-012-1586-7.

Meehl, G. A., J. M. Arblaster, J. T. Fasullo, A. Hu, and K. E. Trenberth, 2011: Model-based evidence of deep-ocean heat 
uptake during surface-temperature hiatus periods. Nat. Climate Change, 1, 360-364, https://doi.org/10.1038/nclimate1229.

—, A. Hu, J. M. Arblaster, J. Fasullo, and K. E. Trenberth, 2013: Externally forced and internally generated decadal climate variability associated with the Interdecadal Pacific Oscillation. J. Climate, 26, 7298-7310, https://doi.org/10.1175/JCLI-D-1200548.1 .

Menary, M. B., D. L. Hodson, J. I. Robson, R. T. Sutton, and R. A. Wood, 2015: A mechanism of internal decadal Atlantic Ocean variability in a high-resolution coupled climate model. J. Climate, 28, 7764-7785, https://doi.org/10.1175/JCLI-D-150106.1.

Middlemas, E. A., and A. C. Clement, 2016: Spatial patterns and frequency of unforced decadal-scale changes in global mean surface temperature in climate models. J. Climate, 29, 62456257, https://doi.org/10.1175/JCLI-D-15-0609.1.

,,-- B. Medeiros, and B. Kirtman, 2019: Cloud radiative feedbacks and El Niño-Southern Oscillation. J. Climate, 32, 4661-4680, https://doi.org/10.1175/JCLI-D-18-0842.1.

Muller, R. A., and Coauthors, 2013: Decadal variations in the global atmospheric land temperatures. J. Geophys. Res., 118, 5280-5286, https://doi.org/10.1002/jgrd.50458.

Newman, M., and Coauthors, 2016: The Pacific decadal oscillation, revisited. J. Climate, 29, 4399-4427, https://doi.org/10.1175/ JCLI-D-15-0508.1.

North, G. R., T. L. Bell, R. F. Cahalan, and F. J. Moeng, 1982: Sampling errors in the estimation of empirical orthogonal functions. Mon. Wea. Rev., 110, 699-706, https://doi.org/ 10.1175/1520-0493(1982)110<0699:SEITEO > 2.0.CO;2.

Oldenburg, D., R. C. J. Wills, K. C. Armour, L. Thompson, and L. C. Jackson, 2021: Mechanisms of low-frequency variability in North Atlantic Ocean heat transport and AMOC. J. Climate, 34, 4733-4755, https://doi.org/10.1175/JCLI-D-20-0614.1.

Olonscheck, D., M. Rugenstein, and J. Marotzke, 2020: Broad consistency between observed and simulated trends in sea surface temperature patterns. Geophys. Res. Lett., 47, e2019GL086773, https://doi.org/10.1029/2019GL086773.

Otto, A., and Coauthors, 2013: Energy budget constraints on climate response. Nat. Geosci., 6, 415-416, https://doi.org/10.1038/ngeo1836.

Palmer, M. D., D. J. McNeall, and N. J. Dunstone, 2011: Importance of the deep ocean for estimating decadal changes in Earth's radiation balance. Geophys. Res. Lett., 38, L13707, https://doi.org/10.1029/2011GL047835.

Pan, Y. H. and and A. H. Oort, 1983: Global climate variations connected with sea surface temperature anomalies in the eastern equatorial Pacific Ocean for the 1958-73 period. Mon. Wea. Rev., 111, 1244-1258, https://doi.org/10.1175/1520-0493(1983) $111<1244:$ GCVCWS $>2.0$.CO;2.

Parsons, L. A., and G. J. Hakim, 2019: Local regions associated with interdecadal global temperature variability in the Last Millennium Reanalysis and CMIP5 models. J. Geophys. Res., 124, 9905-9917, https://doi.org/10.1029/2019JD030426.

— M. K. Brennan, R. C. Wills, and C. Proistosescu, 2020: Magnitudes and spatial patterns of interdecadal temperature variability in CMIP6. Geophys. Res. Lett., 47, e2019GL086588, https://doi.org/10.1029/2019GL086588.

Pedro, J. B., T. Martin, E. J. Steig, M. Jochum, W. Park, and S. O. Rasmussen, 2016: Southern ocean deep convection as a driver of Antarctic warming events. Geophys. Res. Lett., 43, 21922199, https://doi.org/10.1002/2016GL067861.

Philander, S. G., 1989: El Niño, La Niña, and the Southern Oscillation. International Geophysics Series, Vol. 46, Academic Press, 293 pp.
Proistosescu, C., A. Donohoe, K. C. Armour, G. H. Roe, M. F. Stuecker, and C. M. Bitz, 2018: Radiative feedbacks from stochastic variability in surface temperature and radiative imbalance. Geophys. Res. Lett., 45, 5082-5094, https://doi.org/ 10.1029/2018GL077678.

Rädel, G., T. Mauritsen, B. Stevens, D. Dommenget, D. Matei, K. Bellomo, and A. Clement, 2016: Amplification of El Niño by cloud longwave coupling to atmospheric circulation. Nat. Geosci., 9, 106-110, https://doi.org/10.1038/ngeo2630.

Rasmusson, E. M., and T. H. Carpenter, 1982: Variations in tropical sea surface temperature and surface wind fields associated with the Southern Oscillation/El Niño. Mon. Wea. Rev., 110, 354-384, https://doi.org/10.1175/1520-0493(1982) $110<0354$ :VITSST $>2.0$. CO 2 .

Reintges, A., T. Martin, M. Latif, and W. Park, 2017: Physical controls of Southern Ocean deep-convection variability in CMIP5 models and the Kiel Climate Model. Geophys. Res. Lett., 44, 6951-6958, https://doi.org/10.1002/2017GL074087.

Risbey, J. S., S. Lewandowsky, C. Langlais, D. P. Monselesan, T. J. O'Kane, and N. Oreskes, 2014: Well-estimated global surface warming in climate projections selected for ENSO phase. Nat. Climate Change, 4, 835-840, https://doi.org/10.1038/ nclimate2310.

Schmeisser, L., T. Ackerman, and N. Bond, 2020: Marine heatwaves confirm cloud response to warming in global climate models. Earth Space Sci. Open Arch.,https://doi.org/10.1002/ essoar.10503088.1.

Schneider, T., and S. M. Griffies, 1999: A conceptual framework for predictability studies. J. Climate, 12, 3133-3155, https://doi.org/ 10.1175/1520-0442(1999)012<3133:ACFFPS > 2.0.CO;2.

- , and I. M. Held, 2001: Discriminants of twentieth-century changes in Earth surface temperatures. J. Climate, 14, 249-254, https:// doi.org/10.1175/1520-0442(2001)014<0249:LDOTCC >2.0.CO;2.

Sherwood, S., and Coauthors, 2020: An assessment of Earth's climate sensitivity using multiple lines of evidence. Rev. Geophys., 58, e2019RG000678, https://doi.org/10.1029/ 2019RG000678.

Stolpe, M. B., I. Medhaug, and R. Knutti, 2017: Contribution of Atlantic and Pacific multidecadal variability to twentiethcentury temperature changes. J. Climate, 30, 6279-6295, https://doi.org/10.1175/JCLI-D-16-0803.1.

,-- J. Sedláček, and R. Knutti, 2018: Multidecadal variability in global surface temperatures related to the Atlantic meridional overturning circulation. J. Climate, 31, 2889-2906, https://doi.org/10.1175/JCLI-D-17-0444.1.

Stuecker, M. F., and Coauthors, 2018: Polar amplification dominated by local forcing and feedbacks. Nat. Climate Change, $\mathbf{8}$, 1076-1081, https://doi.org/10.1038/s41558-018-0339-y.

Takahashi, K., A. Montecinos, K. Goubanova, and B. Dewitte, 2011: ENSO regimes: Reinterpreting the canonical and Modoki El Niño. Geophys. Res. Lett., 38, L10707, https://doi.org/10.1029/ 2011 GL047364.

Timmermann, A., and Coauthors, 2018: El Niño-Southern Oscillation complexity. Nature, 559, 535-545, https://doi.org/10.1038/s41586018-0252-6.

Ting, M., Y. Kushnir, R. Seager, and C. Li, 2009: Forced and internal twentieth-century SST trends in the North Atlantic. J. Climate, 22, 1469-1481, https://doi.org/10.1175/2008JCLI2561.1.

Trenberth, K. E., and J. T. Fasullo, 2013: An apparent hiatus in global warming? Earth's Future, 1, 19-32, https://doi.org/ 10.1002/2013EF000165.

_ J. J. Caron, D. P. Stepaniak, and S. Worley, 2002: Evolution of El Niño-Southern Oscillation and global atmospheric surface 
temperatures. J. Geophys. Res., 107, 4065, https://doi.org/ 10.1029/2000JD000298.

Tung, K.-K., X. Chen, J. Zhou, and K.-F. Li, 2018: Interdecadal variability in pan-Pacific and global SST, revisited. Climate Dyn., 52, 2145-2157, https://doi.org/10.1007/s00382-018-4240-1.

Venzke, S., M. R. Allen, R. T. Sutton, and D. P. Rowell, 1999: The atmospheric response over the North Atlantic to decadal changes in sea surface temperature. J. Climate, 12, 2562-2584, https://doi.org/10.1175/1520-0442(1999)012<2562:

TAROTN $>2.0 . \mathrm{CO} ; 2$.

Watanabe, M., J.-L. Dufresne, Y. Kosaka, T. Mauritsen, and H. Tatebe, 2021: Enhanced warming constrained by past trends in equatorial Pacific sea surface temperature gradient. Nat. Climate Change, 11, 33-37, https://doi.org/10.1038/s41558-020-00933-3.

Wigley, T., 2000: ENSO, volcanoes and record-breaking temperatures. Geophys. Res. Lett., 27, 4101-4104, https://doi.org/ 10.1029/2000GL012159.

Wills, R. C. J., X. J. Levine, and T. Schneider, 2017: Local energetic constraints on Walker circulation strength. J. Atmos. Sci., 74, 1907-1922, https://doi.org/10.1175/JAS-D-16-0219.1.

—, T. Schneider, J. M. Wallace, D. S. Battisti, and D. L. Hartmann, 2018: Disentangling global warming, multidecadal variability, and El Niño in Pacific temperatures. Geophys. Res. Lett., 45, 2487-2496, https://doi.org/10.1002/2017GL076327.

—, K. C. Armour, D. S. Battisti, and D. L. Hartmann, 2019a: Ocean-atmosphere dynamic coupling fundamental to the Atlantic multidecadal oscillation. J. Climate, 32, 251-272, https://doi.org/10.1175/JCLI-D-18-0269.1.

— D. S. Battisti, C. Proistosescu, L. Thompson, D. L. Hartmann, and K. C. Armour, 2019b: Ocean circulation signatures of North Pacific decadal variability. Geophys. Res. Lett., 46, 1690-1701, https://doi.org/10.1029/2018GL080716.

, K. C. Armour, T. Schneider, and C. Deser, 2020: Pattern recognition methods to separate forced responses from internal variability in climate model ensembles and observations. J. Climate, 33, 8693-8719, https://doi.org/10.1175/ JCLI-D-19-0855.1.

Xie, S.-P., Y. Kosaka, and Y. M. Okumura, 2016: Distinct energy budgets for anthropogenic and natural changes during global warming hiatus. Nat. Geosci., 9, 29-33, https://doi.org/10.1038/ ngeo2581.
Yeager, S. G., A. R. Karspeck, and G. Danabasoglu, 2015: Predicted slowdown in the rate of Atlantic sea ice loss. Geophys. Res. Lett., 42, 10 704-10 713, https://doi.org/10.1002/ 2015 GL065364.

Yuan, T., L. Oreopoulos, M. Zelinka, H. Yu, J. R. Norris, M. Chin, S. Platnick, and K. Meyer, 2016: Positive low cloud and dust feedbacks amplify tropical North Atlantic multidecadal oscillation. Geophys. Res. Lett., 43, 1349-1356, https://doi.org/ 10.1002/2016GL067679.

Zebiak, S. E., and M. A. Cane, 1987: A model El Niño-Southern Oscillation. Mon. Wea. Rev., 115, 2262-2278, https://doi.org/ 10.1175/1520-0493(1987)115<2262:AMENO>2.0.CO;2.

Zhang, L., T. L. Delworth, and L. Jia, 2017: Diagnosis of decadal predictability of Southern Ocean sea surface temperature in the GFDL CM2.1 model. J. Climate, 30, 6309-6328, https:// doi.org/10.1175/JCLI-D-16-0537.1.

Zhang, R., and T. L. Delworth, 2007: Impact of the Atlantic multidecadal oscillation on North Pacific climate variability. Geophys. Res. Lett., 34, L23708, https://doi.org/10.1029/ 2007GL031601.

,-- , and I. M. Held, 2007: Can the Atlantic Ocean drive the observed multidecadal variability in Northern Hemisphere mean temperature? Geophys. Res. Lett., 34, L02709, https:// doi.org/10.1029/2006GL028683.

—, R. Sutton, G. Danabasoglu, Y.-O. Kwon, R. Marsh, S. G. Yeager, D. E. Amrhein, and C. M. Little, 2019: A review of the role of the Atlantic meridional overturning circulation in Atlantic multidecadal variability and associated climate impacts. Rev. Geophys., 57, 316-375, https://doi.org/10.1029/ 2019RG000644.

Zhang, Y., J. M. Wallace, and D. S. Battisti, 1997: ENSO-like interdecadal variability: 1900-93. J. Climate, 10, 1004-1020, https:// doi.org/10.1175/1520-0442(1997)010<1004:ELIV>2.0.CO;2.

Zhou, C., M. D. Zelinka, and S. A. Klein, 2016: Impact of decadal cloud variations on the Earth's energy budget. Nat. Geosci., 9, 871-874, https://doi.org/10.1038/ngeo2828.

,-- , and -2017 : Analyzing the dependence of global cloud feedback on the spatial pattern of sea surface temperature change with a Green's function approach. $J . A d v$. Model. Earth Syst., 9, 2174-2189, https://doi.org/10.1002/ 2017MS001096. 